\title{
Provision of a public good with altruistic overlapping generations and many tribes*
}

\author{
Larry $\operatorname{Karp}^{\dagger}$
}

July 17, 2013

\begin{abstract}
I compare the relative importance of intergenerational altruism and contemporaneous cooperation, in determining equilibrium climate policy, by imbedding an OLG framework with intergenerational altruism into a differential game between nations. The model uses new representations for the aggregate preferences of OLGs with paternalistic or pure altruism, and exponentially distributed or deterministic lifetimes. The Markov perfect equilibrium in the resulting game solves an auxiliary control problem. I obtain analytic results for a model with linear climate damages and numerical results for a model with convex damages. The relative importance of altruism and cooperation depends on model specifics and the equilibrium type.
\end{abstract}

Keywords: Overlapping generations, altruism, time consistency, Markov Perfection, differential games, climate policy

JEL, classification numbers: C73, D62, D63, D64, H41, Q54

*Earlier versions of this paper benefitted from comments by Geir Asheim, Ivar Ekeland, Reyer Gerlagh, Antii Iho, Terry Iverson, Jacek Krawczyk, Derek Lemoine, Armon Rezai, Leo Simon, Christian Traeger, and seminar participants at Berkeley, Santa Barbara, Paris, Vienna, SURED 2012, and the TIGER conference, Toulouse 2013. The usual disclaimer holds. I also thank Reyer Gerlagh and Matti Liski for making available numerical inputs to their 2012 paper.

${ }^{\dagger}$ Department of Agricultural and Resource Economics, University of California, Berkeley, and the Ragnar Frisch Center for Economic Research, email: karp@berkeley.edu 


\section{Introduction}

People must cooperate and must value their descendants, to willingly invest in longlived public goods such as climate protection. Public good investment games may have multiple equilibria. In this case, the relative importance of cooperation and intergenerational altruism, in determining investment, may depend both equilibrium type and model specifics. I study a model combining overlapping generations and a differential game, and then specialize to a climate setting. The overlapping generations setting disentangles patience for one's own future consumption, from altruism toward descendants. The differential game captures contemporaneous rivalry.

Two decades of negotiation show how hard it is for policymakers to cooperate on climate policy. A large game theoretic literature examines contemporaneous cooperation in the provision of public goods in general, and climate protection in particular. This theory underpins suggestions for designing an effective climate agreement (Aldy and Stavins, 2007; Guesnerie and Tulkens, 2008). A recent literature emphasizing the role of discounting in selecting climate policy turns on the proper way to evaluate future utility (Stern, 2006; Nordhaus, 2007, Weitzman, 2007). The UK and France have begun to discount long-lived public projects using lower-than-market rates.

To what extent would the widespread use of low discount rates change climate policy, at current levels of international cooperation? How important is increased international cooperation, at given levels of intergenerational altruism? How should modelers account for differences in intra- and inter-generational discount rates in determining the equilibrium provision of a public good?

The paper makes three types of contributions. The methodological contribution provides a framework for evaluating the relative importance of intra-generational cooperation and intergenerational altruism in the provision of a public good. I also provide a measure of the "most cooperative" steady state that can be sustained, as a function of intergenerational altruism and intra-generational cooperation. The policy contribution takes a step in applying this framework to the climate problem. The lesson a reader takes away from this analysis depends on their views about the plausibility of different assumptions. The analysis makes it possible to understand 
the relation between assumptions and results, but does not, of course, yield a ranking that holds under all circumstances. The theoretical contributions clarify the relations between discount functions induced by agents with exponentially distributed or deterministic finite lifetimes, and between pure and paternalistic altruism.

If people discount their own and their descendants' utility at constant but different rates, then the social planner who aggregates their preferences has non-constant discounting, creating time-inconsistency. Having many rather than a single tribe (countries, in the climate policy context) complicates the policy problem. I study a game with $n \geq 1$ tribes, each with an equal share of the world's fixed population and each with many generations. Tribal members care about their own utility stream and - to the extent that they are altruistic - about their descendants' utility streams.

Within each tribe and at each point in time, a planner aggregates the preferences of agents alive, in her tribe, into those of a representative agent. These tribal representatives have both a tribal and a time index; the game has two dimensions. The planners alive in the same period (one for each tribe) act simultaneously, and the planner of any tribe in any period acts before the future planners. I consider symmetric Markov Perfect Equilibria (MPE) to a symmetric game: actions depend on only the payoff-relevant state variable. If there is a single tribe whose members discount their own and their descendants' utility at different rates, the result is the familiar problem of non-constant discounting (Strotz, 1956; Laibson, 1997). If multiple tribes all have constant discount rates, they play a differential game (Long, 2010; Haurie, Krawczyk and Zaccour, 2012). If there is a single tribe with constant discount rate, the result is a standard control problem.

The baseline OLG model contains "paternalistically altruistic" individuals (Ray, 1987; Andreoni, 1989; Saez-Marti and Weibull, 2005) with exponentially distributed lifetimes (Yaari, 1965; Blanchard, 1985). This agent cares about future generations' utility flows, but does not take into account that the not-yet-born also care about their descendants. A discount factor, with non-constant discount rate, aggregates the welfare of agents in a tribe alive at a point in time.

I compare the baseline model with two variations. The first replaces the paternalistic with the "purely" altruistic agent, one who does take into account the 
fact that her successors also value their own successors' utility flows. In the second variation, paternalistically altruist agents live a known finite amount of time, as in Diamond (1965)'s two period model or in Schneider, Traeger, and Winkler (2012)'s continuous time setting. These variations provide a robustness check for results; they are of intrinsic interest because they show the relation between the simplest and most familiar model and two alternatives.

To assess the relative importance of altruism and cooperation, in determining climate policy, I need to solve the model, not merely describe its equilibrium; this requires functional assumptions. I begin with a "linear-in-state" model, generalizing the climate component in Golosov et al. 2011. Within the class of state-independent policies, I obtain analytic expressions for the unique equilibrium emissions, the supporting tax, and its elasticities. In the scalar version of this model, international cooperation is more important than altruism in determining climate policy. A higher-dimensional version of this model can reverse that conclusion. In general, in a Nash equilibrium an agent's optimal action depends on the actions that other agents take. In the linear-in-state model with state-independent strategies, equilibrium strategies are dominant: an agent's optimal decision does not depend on the state-independent actions that other agents take. The functional assumptions thus eliminate strategic interactions across generations and nations.

I need a model with convex damages, or some other source of non-linearity, to generate non-trivial strategic interactions. I therefore turn to a linear-quadratic model, and use numerical methods. ${ }^{1}$ The linear equilibrium to this model (like the state-independent equilibrium in the linear-in-state model) is the limit of the equilibria of the finite horizon model, as the horizon becomes large. There are many other equilibria in the infinite horizon setting, even with Markov perfection and requiring differentiability. For the linear equilibrium, cooperation is again more

\footnotetext{
${ }^{1}$ The linear-quadratic constant-discounting differential game has been widely used in both industrial organization (Fershtman and Kamien, 1987; Reynolds, 1987) and natural resource economics (van der Ploeg, 1992; Dockner and Long, 1993; Wirl, 1994); it has also been used to study quasihyperbolic discounting in the one-agent setting (Karp 2005). A log-linear model (logarithmic utility and Cobb Douglas growth functions) is a popular alternative, both for differential games (Levhari and Mirman 1980) and for non-constant discounting (Barro 1999).
} 
important than altruism. However, this conclusion is easily reversed if we also consider equilibria arising only in the infinite horizon setting. The life of our planet is finite, but equilibria that rely on an infinite horizon can be motivated as $\varepsilon$-equilibria to a finite horizon game (Fudenberg and Levine 1983).

I obtain a continuous time setting by taking the limit of a discrete time model as the length of a period becomes small. This approach makes the mathematics straightforward. Technical details appear in appendices.

\section{Discounting}

The objective of this paper is to evaluate the relative influence, on investment in a public good, of agents' attitudes toward future generations and on the ability of different groups to cooperate at a point in time. Infinitely-lived- agent models conflate impatience with respect to one's own future utility and attitudes toward descendents' utility. An OLG model is essential for disentangling these two distinct preference attributes. A discount function aggregates the intertemporal preferences of different generations within a tribe. I emphasize the case where lifetime is exponentially distributed and agents' altruism is paternalistic, and then consider two alternatives. The first replaces paternalistic with pure altruism, under exponentially distributed lifetime. The second alternative replaces the exponentially distributed lifetime with a known finite lifetime, under paternalistic altruism. Several papers use a convex combination of exponentials to represent non-constant discounting ( $\mathrm{Li}$ and Lofgren, 2000; Gollier and Weitzman, 210; Zuber, 2010; and Jackson and Yariv, 2011). Ekeland and Lazrak (2010)'s motivation, based on an OLGs, is quite different, and leads to a sum (but not necessarily convex combination) of exponentials. Their paper provides the formula for exponentially distributed lifetime with paternalistic altruism (equation (1), below), but the two alternatives are new.

The population is constant, so the birth rate equals the death rate. This section constructs and analyzes the discount factor to aggregate utility streams. Consider a public project, e.g. protection of the climate system. An agent's utility flow at a point in time depends on the current stock of the public good (e.g., greenhouse 
gasses, GHGs) and on her tribe's current investment in that good (e.g., abatement). This investment cost is shared equally by all tribal members then alive, so at time $t$ they all have the same utility flow, $u_{t}{ }^{2}$ For an arbitrary sequence of bounded utility flows, $\left\{u_{\tau}\right\}_{\tau=t}^{\infty}$, I find the discount factor $D(\tau)$ for which welfare of a social planner at $t$ is $\int_{t}^{\infty} D(\tau) u_{\tau} d \tau$. The discount factor $D(\tau)$ depends on the both the type of altruism and on whether lifetime is exponentially distributed or deterministic. There is no tribal index here, because I consider a representative tribe.

\subsection{Exponential lifetime, paternalistic altruism}

Agents' welfare consists of a selfish and an altruistic component. The selfish component equals the expected present discounted value of the flow of the agent's utility, using a constant pure rate of time preference, $r$. The altruistic component consists of the agent's evaluation of her successors' stream of utility. Those successors discount their own utility at the constant rate $r$, and the previous agent discounts the selfish component of her successors' welfare at rate $\lambda$. Completely selfish agents put no weight on the welfare of the unborn $(\lambda=\infty)$. A smaller value of $\lambda$ implies a higher level of altruism. "Altruism" here refers to benevolence toward one's descendants, not toward other tribes. At the cost of introducing another parameter, one could distinguish between altruism within and across tribes. The paternalistic agent cares about her successors' utility stream, but does not take into account that each successor also cares about their own successors' utility stream. The agent's mortality rate is $\theta$, so $\gamma \equiv r+\theta$ is the agent's risk-adjusted pure rate of time preference. Measure zero individuals face mortality risk, but there is no aggregate risk.

The memoryless feature of the exponential distribution means that all tribal members alive at a point in time have the same distribution function for their remaining lifetime. Because there is no private capital accumulation in this model, all agents alive at a point in time are identical. In this case, there is a representative agent in

\footnotetext{
${ }^{2}$ Schneider et al (2012) note that the relation between the intergenerational discount rate and the selfish pure rate of time preference affects the distribution, amongst agents living at a point in time, that a planner would like to achieve. In order to focus on intergenerational allocations, I exclude the possibility of such intra-generational transfers.
} 
the usual sense. The discount factor for this agent, and thus the discount factor for the social planner who represents these identical agents, is

$$
D(t)=\left(\frac{\lambda-r}{\lambda-\gamma}\right) e^{-\gamma t}-\frac{\theta}{\lambda-\gamma} e^{-\lambda t}
$$

\subsection{Alternative OLG models}

Both because of its intrinsic interest, and also to gauge the sensitivity of the results to assumptions about agents' lifetime and their type of altruism, I consider two alternatives to the model above.

\subsubsection{Pure altruism, exponentially distributed lifetime}

The purely altruistic agent does take into account the fact that her successors care about their own successors' utility streams. Deriving the discount factor for the representative agent under pure altruism is more complicated than under paternalistic altruism. In the latter case, one can simply write down the discount factor from its definition, and then simplify by changing the order of an integration to obtain equation (1). With pure altruism, in contrast, it is necessary to solve a recursion. I achieve this in two stages. First, I begin with a discrete time model, in which each period lasts for $\varepsilon$ units of time. I solve the resulting discrete time recursion, to obtain the discrete time discount function for the representative agent. Taking the limit as $\varepsilon \rightarrow 0$ gives the continuous time discount function under pure altruism. Comparing that function with the expression for $D(t)$ in equation (1) establishes an isomorphism between paternalistic and pure altruism for individual agents, and thus for the representative agent of a tribe:

Proposition 1 Suppose the agent with pure altruism discounts future agents' welfare at rate $\lambda^{\prime}$, and the agent with paternalistic altruism discounts future agents' utility at rate $\lambda$. Both have exponentially distributed lifetimes with mortality rate $\theta$ and the pure rate of time preference $r$. The two agents have the same preferences if and only if $\lambda^{\prime}=\lambda+\theta$. 
The following corollary is a consequence of Proposition 1 and the fact that for $D(t)$ given by equation (1), $\frac{d D}{d \lambda}<0$ for $t>0$ :

Corollary 1 Given the same mortality rate, $\theta$, and the same discounting preference parameters $(r, \lambda)$, the agent with paternalistic altruism discounts the future flow of utility more heavily than the agent with pure altruism.

This comparison is not surprising: the agent with pure altruism cares about future utility flows both because they affect the future generations that directly experience those flows, and because they affect the welfare of earlier generations that care about those future generations. In contrast, the agent with paternalistic altruism cares only about the direct affect of future utility flows on the agents who experience them.

\subsubsection{Paternalistic altruism, known finite lifetime}

Each agent lives for $T=\frac{1}{\theta}$ years, so the known finite lifetime in this setting equals the expected lifetime in the exponentially distributed setting: the two models are directly comparable. With deterministic lifetimes, agents alive at a point in time are different: the older ones will die sooner than the younger ones. In this setting, I assume that the social planner at a point in time is utilitarian; she puts equal weight on the preferences of all tribal members currently alive. This model can be viewed as a generalization of the $\beta, \delta$ of quasi-hyperbolic discounting (Laibson 1997). That relation is easiest to see in a discrete time setting; see Appendix A.1.3

The structure of the discount function differs for $t<T$ and $t>T$. For $t<T$, some of the agents alive at time 0 will still be alive. Those agents continue to benefit from the utility flow, and that utility flow contributes to the selfish component of the welfare of the time 0 social planner. For $t>T$, all of the agents alive at time 0 will have died, so the social planner at time 0 places a positive weight on the utility flow at $t>T$ only to the extent that agents are altruistic $(\lambda<\infty)$. Calculations establish:

Proposition 2 For finitely lived agents, the discount factor is 


$$
D(t)=\left\{\begin{array}{c}
e^{-r t}\left(\frac{1-e^{-(\lambda-r) t}}{T(\lambda-r)}+\frac{T-t}{T}\right) \text { for } t \leq T \\
e^{-\lambda t}\left(e^{(\lambda-r) T}-1\right) \frac{1}{T(\lambda-r)} \text { for } t \geq T .
\end{array}\right.
$$

\subsection{Discount rates: a comparison}

The model of exponentially distributed lifetime is more tractable and more frequently used, but perhaps less descriptive than the model of finitely lived agents. Comparison of the two models with paternalistic preferences shows how the assumptions about lifetime affect the imputed discount factors, and thus influence model results. The discount rate, $\eta$, for the baseline model with discount factor in equation (1) is

$$
\eta(t) \equiv-\frac{d D}{d t} \frac{1}{D}=\frac{-\gamma \lambda+\gamma r+\theta \lambda e^{-(\lambda-\gamma) t}}{-\lambda+r+\theta e^{-(\lambda-\gamma) t}}
$$

and the discount rate for the agent with finite lifetime, using equation (2), is

$$
\eta(t)=\left\{\begin{array}{c}
\frac{\lambda e^{-(\lambda-r) t}-r(\lambda-r)(T-t)-\lambda}{e^{-(\lambda-r) t}-1-(\lambda-r)(T-t)} \text { for } t \leq T \\
\lambda \text { for } t \geq T .
\end{array}\right.
$$

Table 1 summarizes the characteristics of the discount rates. If agents discount future generations' utility less than their own $(\lambda<r)$, the discount rate falls from the selfish rate $r$ to $\lambda$; for $\lambda=r$, the discount rate is constant.

\begin{tabular}{|l|l|l|l|l|}
\hline & $0 \leq \lambda<r$ & $\lambda=r$ & $r<\lambda<\infty$ & $\lambda=\infty$ \\
\hline \multicolumn{5}{|c|}{ Lifetime is exponentially distributed } \\
\hline$\frac{d \eta}{d t}$ & - & 0 & + & 0 \\
\hline$\eta(0)$ & $r$ & $r$ & $r$ & $r+\theta$ \\
\hline$\eta(\infty)$ & $\lambda$ & $r$ & $r+\theta$ & $r+\theta$ \\
\hline \multicolumn{5}{|c|}{ Lifetime is finite and deterministic } \\
\hline$\frac{d \eta}{d t}$ for $t<T$ & - & 0 & + & + \\
\hline$\eta(0)$ & $r$ & $r$ & $r$ & $r$ \\
\hline$\eta(t)$ for $t \geq T$ & $\lambda$ & $r$ & $\lambda$ & $\lambda$ \\
\hline
\end{tabular}

Table 1: Characteristics of discount rates 


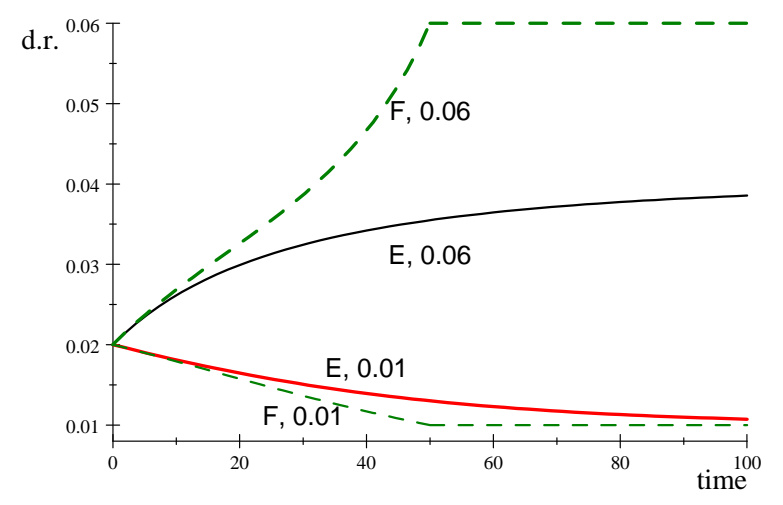

Figure 1: Discount rates (d.r.) for $\theta=0.02=r=\frac{1}{T}$. Solid curves (labelled E) correspond to exponentially distributed lifetime and dashed curves (labelled F) correspond to fixed lifetime. Numerical values in label show value of $\lambda$.

If agents have less concern for future generations' welfare than for their own, $(r<\lambda)$, the discount rate increases over time from $r$; with exponentially distributed lifetime, the discount rate increases to $r+\theta$ as $\lambda \rightarrow \infty$, and with finite deterministic lifetime, the discount rate increases to $\lambda$. With exponentially distributed lifetime, the probability that an agent is alive at a point in time in the future decreases at a constant rate. Therefore, even if she cares nothing about unborn generations, her discount rate for future utility flows never rises above $r+\theta$. In the deterministic lifetime case, all agents currently alive will be dead within $T$ years, so the utilitarian social planner who aggregates their preferences values utility flows after $T$ years only to the extent that agents currently alive care about future generations.

Figure 1 illustrates the discount rates in the two models, using $r=0.02=\theta$ (so $T=50$ ). Solid curves (labelled $E$ ) correspond to exponentially distributed lifetime, and dashed curves (labelled $F$ ) correspond to fixed lifetime. The increasing curves correspond to $\lambda=0.06$ and the decreasing curves correspond to $\lambda=0.01$.

In summary, the discount rate trajectories for the two models have the same initial value, the same direction of change over time, and for $\lambda \approx r+\theta$ or smaller, they have similar long run values: the two trajectories are similar. However, if $\lambda>r+\theta$ the two discount rates are quite different for $t>T$; this difference is 
likely to be important for current decisions unless $T$ is large. Thus, unless $\lambda$ is large and $T$ is small, the trajectories of discount rates, and thus preferences, under the two models are "quite similar". The actual difference between results based on the two assumptions about lifetime depends on model specifics (Section 4.2.2).

The formulae for the discount rates also show the importance of the OLG structure. If $\theta=\infty(T=0)$ a tribe consists of a succession of agents, each of whom lives for a single instant, and has a constant discount rate $\lambda$. At the other extreme, $\theta=0$ $(T=\infty)$ a tribe consists of an infinitely lived agent, with a constant discount rate $r$. For these two limiting cases, there is no time consistency problem.

\section{The game}

I use equilibrium conditions obtained by taking the limit, as the length of a period goes to 0 , of the equilibrium conditions to a discrete stage game. In that game, social planner $i, t$ has a tribal index $i$ and a time index $t$; this planner takes an action, $x_{i t}$. The value of the state variable at $t$, common to all tribes, is $S_{t}$. A stationary symmetric MPE is a function $\chi(S)$ with the Nash property: if planner $i, t$ believes that all future planners (including those in her own tribe) and all other planners currently alive, will make their decision according to $x=\chi(S)$ (where $S$ is the value of the state when a particular decision is made) then it is optimal for planner $i, t$ to set $x_{i t}=\chi\left(S_{t}\right)$. (I use "believe" to mean "act as if she knows".) Planner $i, t$ chooses a single state-contingent action, rather than a (possibly infinite) sequence of actions, as in a control problem. A Nash equilibrium requires that her action maximize her current flow payoff plus a state-dependent continuation value.

Karp (2007), building on Harris and Laibson (2001), finds the formal limit, as the length of a period goes to 0 , of the equilibrium conditions to the discrete time sequential game where $n=1$. Ekeland and Lazrak (2010) independently obtain the same necessary conditions using a variational argument that begins with a continuous time model, i.e., without detouring via the discrete time game. The symmetry assumption makes it possible to use those necessary conditions for the game with $n>1$, making only minor changes involving notation. The succession of planners in 
tribe $i$ who believe that all planners in all other tribes will use the policy rule $\chi(S)$ face the problem considered in Karp (2007) and Ekeland and Lazrak (2010), apart from details regarding notation, described below. For the climate model, I establish existence constructively.

Denote $\mathbf{x}_{t} \in \mathbb{R}^{n}$ as the vector of actions at time $t$, with $i$ 'th element $x_{i t}$, the action taken by planner $i, t$. In the continuous time limit, the state variable $S$ evolves according to $\frac{d S}{d t}=f\left(S_{t}, \mathbf{x}_{t} ; n\right)$, with the initial condition (the current value of $S$ ) given. For example, $S_{t}$ is the stock of GHG, with $x_{i t}$ equal to tribe $i$ 's emissions at time $t$. Tribe $i$ 's flow of utility at $t, u\left(S_{t}, x_{i t} ; n\right)$, depends only on the state variable and $i$ 's action; including $j$ 's current action in $i$ 's utility flow would make it possible to consider trade effects and cross-tribal altruism.

An increase in the parameter $n$ in the growth function, $f$, and the utility function, $u$, represents a fragmentation of the economy that leaves unchanged the set of feasible utility. Fragmentation (a larger $n$ ) has no intrinsic effect on aggregate (or per capita) utility or stock changes, but it does alter the equilibrium decisions, thereby altering the equilibrium aggregate utility and stock changes: $n$ has a strategic but not an intrinsic effect on outcomes. For example, if the world consists of $N$ countries, and each tribe controls $m$ countries, then $n=\frac{N}{m}$. A tribal social planner internalizes the effect of her action on residents in all $m$ of the countries that the tribe controls. If each of these tribes fragments into two equal tribes, then $n=2 \frac{N}{m}$. It is worth emphasizing that a larger $n$ means not only that there are more tribes, but also that each tribe is absolutely smaller (not merely a smaller fraction of the whole). ${ }^{3}$

Denote $\mathbf{i}_{n-1} \in \mathbb{R}^{n-1}$ as the vector consisting of 1's. Given the decision rule $\chi$ that planners in tribe $i$ expect planners in other tribes to use, denote $F\left(S, x_{i}\right) \equiv$ $f\left(S, \mathbf{i}_{n-1} \chi(S), x_{i} ; n\right)$. This function is the time derivative of the stock at a point in time when the current value of the state variable is $S$, all other tribes use $\chi(S)$, and

\footnotetext{
${ }^{3}$ When members of the EU allow a central agent to determine their climate policy, that agent has the incentive to internalize a greater fraction of the effect of a single country's emissions, than would the country. Apart from the fact that countries in the real world are not identical, a coalition such as the EU is analagous to a smaller $n$.
} 
tribe $i$ uses $x_{i}$. The payoff and constraint facing tribe $i$ is

$$
\int_{\tau}^{\infty} D(t-\tau) u\left(S_{t}, x_{i t} ; n\right) d t \quad \text { and } \quad \dot{S}_{t} \equiv \frac{d S_{t}}{d t}=F\left(S, x_{i}\right) .
$$

The discount factor, $D$, is given by equation (1) if agents have exponentially distributed lifetime, and by equation (2) if agents have deterministic lifetime.

A larger value of $n$ means that the time $t$ social planner in tribe $i$ internalizes a smaller fraction of the effect of her actions on other tribes. A larger value of $\lambda$ means that this social planner internalizes a smaller fraction of the future effect of her actions. The point of this paper is to determine the relative sensitivity of equilibrium investments in a public good, to changes in $n$ and $\lambda$.

\subsection{Equilibrium conditions}

Given beliefs that all planners in other tribes use the decision rule $\chi(S)$, equation (5) contains the payoff and constraint facing tribe $i$ in the continuous time limit of a discrete time game. I use the conditions given in Karp (2007) to obtain the necessary conditions to that game. The symmetric Nash condition requires that the equilibrium policy function equals $\chi(S)$ : this function is a fixed point. The validity of this procedure requires that the value function, defined below, and its first derivative exist - an assumption that can be checked given a particular equilibrium.

The equilibrium conditions differ in the two cases corresponding to $\lambda\langle r$ and $\lambda>$ $r$ with exponentially distributed lifetime (because $\lim _{t \rightarrow \infty} \eta(t)$ differs in these two cases), and in the case where agents have deterministic lifetimes (because the function $K$, introduced below, has a different form here). For $\lambda=r$ with both deterministic and exponentially distributed lifetimes, and for $\lambda=\infty$ with exponentially distributed lifetime, the discount rate is constant. In these cases, the tribes play a standard differential game, i.e. one without the strategic interactions within a tribe, across periods. I provide details for the model with exponentially distributed lifetime and $0<\lambda \leq r$ (where $\lim _{t \rightarrow \infty} \eta(t)=\lambda$ ), relegating the other two cases to Appendix B.1.

Dropping the tribal index $i$ (because of symmetry) Proposition 1 and Remark 
1 of Karp (2007) imply that $\chi(S)$ satisfies the necessary condition to the following auxiliary optimal control problem with constant discount rate $\lambda$ :

$$
J(S)=\max \int_{0}^{\infty} e^{-\lambda t}\left(u\left(S_{t}, x_{t} ; n\right)-K\left(S_{t}\right)\right) d \tau \quad \text { subject to } \dot{S}=F(S, x),
$$

with the side condition (a definition):

$$
K\left(S_{t}\right)=(r-\lambda) \int_{0}^{\infty} e^{-\gamma \tau} u\left(S_{t+\tau}^{*}, \chi\left(S_{t+\tau}^{*}\right) ; n\right) d \tau .
$$

The tribe's utility flow on the equilibrium path is $u\left(S_{\tau}, \chi\left(S_{\tau}\right) ; n\right)$, and $S_{\tau}^{*}$ is the solution to the differential equation in (6) when all agents use the decision rule $\chi(S)$. The function $K$ can be interpreted as an annuity, which if received in perpetuity and discounted at the rate $r-\lambda$, equals the present value of the stream of future utility, discounted at the rate $\gamma=r+\theta{ }^{4}$

This model includes familiar special cases. For $n>1$, the endogenous function $F(S, x)=f\left(S, \mathbf{i}_{n-1} \chi(S), x ; n\right)$ depends on the policies of the other $n-1$ agents. Those agents do not exist if $n=1$, in which case, $F(S, x)=f(S, x ; 1)$, an exogenous function, and the model collapses to a sequential game with a single agent at each point in time. For $\lambda=r, K \equiv 0$ and the model collapses to a standard (constant discounting) differential game for $n>1$ or a control problem for $n=1$.

\subsection{Nonuniqueness}

In general, the equilibrium to this game is not unique. Tsutsui and Mino (1990) note the existence of a continuum of stable steady states (an open interval) in the differential game with constant discounting when decision rules are differentiable. For each point in this interval there is an equilibrium policy function, defined at least in the neighborhood of that point. The economic explanation for this multiplicity

\footnotetext{
${ }^{4}$ The dynamic programming equation is $\lambda J(S)=\max _{x}\left[u\left(S_{t}, x_{t} ; n\right)-K\left(S_{t}\right)+J_{S}(S) F(S, x)\right]$. In the two models of Section $4, u$ is concave and $F$ is linear in $x$, so a sufficient condition for equilibrium is that $\chi(S)=\arg \max \left[u\left(S_{t}, x_{t} ; n\right)+J_{S}(S) F(S, x)\right]$ and $J$ solves the DPE. The sufficient conditions for a MPE are simpler in the game here, involving non-constant discount rates, than in the differential game with constant discount rates. Appendix B.2 discusses this issue.
} 
in the differential game is that the decision whether to remain in a particular steady state depends on an agent's beliefs regarding the actions that rivals would take if a single agent were to drive the state away from that steady state. The MPE conditions do not pin down these beliefs. In a standard optimal control problem, the envelope theorem eliminates that kind of consideration, because the first order welfare effect of a deviation from the steady state is 0 . This theorem is not applicable in the differential game, because rivals' actions do not maximize an agent's welfare. The same consideration applies in $n=1$ under non-constant discounting.

When $n>1$ and the discount rate is non-constant, there are two sources of multiplicity of steady states, so the equilibrium is unique (within the class that induce differentiable value functions) only under special circumstances (Ekeland, Karp, and Sumaila 2012). The multiplicity of equilibria creates a coordination problem across tribes and generations. ${ }^{5}$ Some MPE may Pareto dominate others.

\subsection{Interpretation of this game}

My objective is to provide intuition about how limited cooperation amongst contemporaneous agents, and different levels and types of intergenerational altruism, interact to affect equilibrium decisions. Here I consider the paternalistic agent with exponentially distributed lifetime. For $n=1$, there is no conflict amongst contemporaneous agents, and for $\lambda=r$ or $\lambda=\infty$ the time inconsistency problem also vanishes. These parameter values produce a standard optimization problem, with the usual normative interpretation, instead of a game, whose equilibria typically do not have normative properties.

The lower is $\lambda$, the more weight people today put on both the utility flows and the welfare of their successors; thus, $\lambda$ is an inverse measure of altruism. In this stationary model, the upper bound of altruism corresponds to $\lambda=0$. This definition

\footnotetext{
${ }^{5}$ If one drops the assumption that the value function is differentiable, many other MPE can exist (Dutta and Sundaram 1993). Agents might "behave well" if the state variable is in a certain region, but follow a "bad" MPE if the state variable leaves that region. This kind of MPE has the flavor of trigger strategies in repeated games. There are many types of equilibria, apart from the MPE with differentiable value functions, studied here. Krusell and Smith (2003) and Vieille and Weibull (2009) discuss multiplicity in different settings.
} 
of altruism is consistent with different conclusions about what constitutes an ethical choice of $\lambda$. The utility flow $t$ periods in the future may be shared by some agents currently alive and by some agents who have not yet been born. The agent currently alive puts the same weight on the utility flow received by her future self and the notyet born if and only if $\lambda=r$. If ethical preferences require equal weighting of utility, across agents with different ages, then $\lambda=r$ would be regarded as ethical. With this view, the model has a standard normative interpretation if $n=1$ and $\lambda=r$.

However, many economists define ethics by comparing welfare rather than pointwise utility flows. For any $\lambda>0$, the agent alive today puts lower value on the lifetime welfare of an agent born in the future, the further in the future that agent is born. An agent weights the present discounted value of the stream of utility of all tribal members equally, (i.e., regardless of their date of birth) only if $\lambda=0$. Basing ethics on welfare rather than utility flows implies that $\lambda=0$ is an ethical choice (Ramsey 1928). ${ }^{6} \quad$ Nevertheless, $\lambda=0$ with $n=1$ implies non-constant discounting and therefore produces a game across generations. An equilibrium outcome to that game is in general not normative, for the same reason that the non-cooperative Nash equilibrium to virtually any game is not normative.

For $\lambda=0$, the discount rate converges to 0 , so unless the flow payoff converges to 0 sufficiently rapidly, the payoff is unbounded. Nevertheless, the equilibrium policy function may be well defined even as $\lambda \rightarrow 0$, as in Section 4 . For small positive $\lambda$ and bounded $u_{i t}$, the payoff is well defined and is approximately proportional to $\frac{u_{\infty}}{\lambda}$, the steady state utility flow divided by $\lambda$ :

Lemma 1 For any bounded utility flow $u(t)$ that converges to $u_{\infty} \neq 0$, and given the discount factor under paternalistic altruism where lifetime is exponentially distributed (equation (1)), $\lim _{\lambda \rightarrow 0}\left(\frac{\lambda}{u_{\infty}} \int_{0}^{\infty} D(t) u(t) d t\right)=\frac{\theta}{\gamma}$.

\footnotetext{
${ }^{6}$ People might object to $\lambda<r$ as implausible rather than unethical: why would an agent today put higher weight on her successor's utility flows than on her own, when they are both alive at a point in time, and both have the same utility flows? That objection amounts to the claim that we can only expect people to base ethical judgements on utility rather than welfare comparisons. In addition, the claim that $\lambda<r$ is implausible may be incorrect. As noted in the Introduction, some countries have decided to discount some public projects at lower than the market-based social discount rate. There may be reasons, having nothing to do with ethics, for this decision; but one rationale for it is to reflect a more ethical treatment of future generations.
} 
For small $\lambda$, the payoff in the steady state determines the evaluation of welfare.

Denote the steady state that maximizes the steady state utility flow as the "Utility Maximizing Steady $S$ tate", or UMSS, the solution to $\max _{S} u(S, x ; 1)$ subject to $f(S, x ; 1)=0$. This is the steady state chosen by the infinitely patient planner who controls the system $(n=1)$; no sensible welfare criterion would choose a smaller steady state (in the case where the state is a bad rather than a good). Suppose that this static optimization problem is concave, so that levels of the state variable closer to the UMSS have higher utility levels. Consider the case where $\lambda$ is small and where the MPE state trajectory does not approach the UMSS. In this situation, Lemma 1 together with the concavity assumption imply that a deviation from the equilibrium that causes the state to move closer to the UMSS, is a Pareto improvement over the MPE. Each generation prefers this deviation.

The following proposition states that for small $\lambda$, there is a MPE that supports a steady state arbitrarily close to the UMSS.

Proposition 3 Consider the class of differentiable MPE policy rules. For $n=1$ and for arbitrarily small positive $\varepsilon$, it is possible to support a MPE steady state that leads to a utility flow within $\varepsilon$ of the utility level at the UMSS, provided that $\lambda$ is sufficiently small (but positive).

This proposition, together with Lemma 1 and the generic multiplicity of equilibria, implies that when $\lambda$ is small but positive, there exists a MPE that maintains the state close to the UMSS; moreover, that MPE Pareto dominates any MPE that maintains the state further from the UMSS. The proof of the proposition uses the assumption that the policy function is differentiable. Section 3.2 notes that for this class of policy function, the set of MPE stable steady states is an open interval. Proposition 3 states that a boundary of that interval moves close to the UMSS as $\lambda$ becomes small. We have no information about the measure of the domain of a policy function that drives the state close to this utility-maximizing level. If that measure is small, it is possible to support such a steady state only for initial conditions close to it. In that case, the existence of this steady state may have little practical importance. 


\section{An application to climate policy}

I begin with a linear-in-state model with state-independent strategies, yielding analytic results. In this model, there are no (interesting) strategic interactions amongst agents, because strategies are dominant. I then consider a linear-quadratic model, which requires numerical methods but gives rise to strategic interactions.

Technological progress may make distant generations vastly richer than us, causing actions that we take over the next several generations to affect successors' welfare for a few generations, but not in the long run: the climate problem ends, perhaps in a millennium or two. With this view, an economic-climate model must account for growth associated with technological change.

For at least three reasons, it is worth considering alternatives to the hypothesis that in the very long run we will grow our way out of the climate problem. First, the familiar relation between high expected growth and high discount rate arises in the standard model with additive expected utility. In a model that disentangles risk aversion from the elasticity of intertemporal substitution, stochastic growth might have little effect on the certainty equivalent discount rate (Traeger 2012b). Second, the assumption, adopted by most integrated assessment models (IAMs), that natural and man-made capital are highly substitutable, may be incorrect (Guersnerie 2004), (Hoel and Sterner 2007), (Traeger 2012a). Third, most IAMs, identify growth with increased GDP. The limitations of GDP as an index (or sole argument) of utility are well understood; alternatives, such as Genuine Progress Indicator (GPI), Human Development Index (HDI) and Ecological Footprint have been proposed as alternatives or supplements. Kubiszewski et al. (2013) find that over the past 25 years, GPI has been flat, while GDP has continued to grow. For these reasons, and in the interest of tractability, I abstract from growth. (But see footnote 7.)

\subsection{The linear-in-state model}

Here I assume that lifetime is exponentially distributed and $\lambda \in[0, r]$, so I use the formulae in Section 3.1; the same methods apply if $\lambda>r$ and for other discount functions. The state variable, $S \in \mathbb{R}^{m}$, includes carbon stocks in different sinks and 
a measure of damages. Gerlagh and Liski (2012) show that a multi-dimensional linear model can capture delayed and persistent emissions' impacts, and can closely mimic damages in DICE (Nordhaus 2008). The flow payoff depends directly on only the first element of $S$ ("damages"), denoted $s \equiv i_{1}^{\prime} S$, where $i_{1}$ is the first unit vector. Primes denote transpose. Appendix B.4.1 confirms claims made in this section.

The equation of motion is $\dot{S}=c+B S+C(x+(n-1) \chi)$. The scalar $x$ is tribe $i^{\prime}$ s emissions, and $\chi$ is the emission of any other tribe, all of which are equal in a symmetric equilibrium. The constant matrix $B$ and vectors $c$ and $C$ determine the climate dynamics and damages. The matrix of eigenvalues of $B$ is $\Lambda$ with $i$ 'th diagonal element $\Lambda_{i}$, and the matrix of eigenvectors is $P$. I assume that $\Lambda_{i}$ are nonpositive real numbers and $P$ is of full rank. If $x$ and $\chi$ (the emissions of a particular tribe, and of all other tribes) are state-independent, but possibly time varying, then $S_{t}=P e^{\Lambda t} P^{-1} S_{0}+H(t) ; H(t)$ depends on values of $x, \chi$ over $(0, t)$.

Suppressing time subscripts, denote $X=n x$ as aggregate emissions in a symmetric equilibrium and $S$ as the state variable at a point in time. Aggregate utility flow is $u(X, s ; 1)=v(X ; 1)-\kappa s$ for a concave function $v ; \kappa$ is the damage parameter. Because $n$ measures fragmentation, not population, aggregate utility in a symmetric equilibrium depends only on aggregate emissions and the state, not explicitly on $n$. Therefore, for the linear-in-state model, utility of a particular tribe is $u(x, s ; n)=v(x ; n)-\frac{\kappa}{n} s$ with $v(x ; n)=\frac{1}{n} v(n x ; 1)$.

Example 1 (Log-linear model) Emissions, $x$, are proportional to fossil fuel use; the elasticity of output with respect to fossil fuel use is $\alpha$, a constant; and utility is logarithmic in consumption = output. Climate-related damage reduces output by the factor $\left(1-e^{-\kappa s}\right)$. With aggregate emissions $X=n x$, aggregate output is $X^{\alpha} e^{-\kappa s}$ and aggregate utility is $\ln \left[(n x)^{\alpha} e^{-\kappa s}\right]$. A tribe emitting $x$ obtains utility $u(x, s ; n)=$ $\frac{1}{n} \ln \left[(n x)^{\alpha} e^{-\kappa s}\right]$. Here, $v(x ; n)=\frac{\alpha}{n} \ln (n x)$ and $v(X ; 1)=v(n x ; 1)=\alpha \ln n x$.

Example 2 (Quadratic model) Utility for a tribe emitting $x$ is $u(x, s ; n)=v(x ; n)$ $\frac{\kappa}{n} s$, with $v(x ; n)=a x-\frac{d n}{2} x^{2} . \quad$ Aggregate utility in a symmetric equilibrium is $n u(x, s ; n)=v(n x ; 1)-\kappa s$, where $v(n x ; 1)=v(X ; 1)=a X-\frac{d}{2} X^{2}$. 
For both examples, aggregate utility and the change in the stock depend on aggregate emissions and the stock, but not directly on $n$. Example 1 is taken from Golosov et al. (2011), eliminating the investment decision. ${ }^{7}$

Proposition 4 For the linear-in-state model, suppose that a planner of a tribe at a point in time believes that all other agents (future planners in her own tribe and all planners in all other tribes) will use state-independent (but possibly time- and tribedependent) emissions policies. (i) Her annuity function, $K(S)$ and value function $J(S)$ are linear in the state, $K(S ; t)=q_{0 t}+q^{\prime} S$ and $J(S ; t)=g_{0 t}+g^{\prime} S$ with:

$q^{\prime}=-\kappa \frac{r-\lambda}{n} \tilde{q}^{\prime}$ with $\tilde{q}^{\prime}=\int_{0}^{\infty} i_{1}^{\prime} P e^{-(\gamma I-\Lambda) \tau} P^{-1} d \tau ; g^{\prime}=\frac{\kappa}{n}\left(i_{1}^{\prime}-(r-\lambda) \tilde{q}^{\prime}\right)(B-\lambda I)^{-1}$,

where $I$ is the $m$ dimensional identity matrix.

(ii) Her optimal action, a constant emissions level, is a dominant strategy: it does not depend on her beliefs about the state-independent actions of any future planner, or about the actions of other current planners. Within the class of state-independent policies, the unique equilibrium is the constant

$$
\chi=\arg \max _{x} v(x ; n)+g^{\prime} C x .
$$

Corollary 2 In the log-linear model, equilibrium emissions per tribe are independent of $n$, so aggregate emissions are proportional to $n$. In the quadratic model, equilibrium emissions per tribe fall with $n$, and aggregate emissions are a strictly concave increasing function of $n$.

For the log-linear model, if each of $n$ tribes fragments into two, aggregate equilibrium emissions double, despite no fundamental (i.e., non-strategic) change in the economy. The quadratic model does not have this extreme feature. The corollary also shows that the comparative statics of emissions depend on the function $v$.

\footnotetext{
${ }^{7} \mathrm{I}$ could replace the production function $X^{\alpha} e^{-\kappa s}$ with $A_{t} X^{\alpha} e^{-\kappa s}$ to capture changes in technology and capital stock. With Cobb Douglas production and logarithmic utility, equilibrium investment is a constant fraction of output, and that constant is independent of climate parameters. In this case, consumption is 1 minus the savings rate times output, instead of output. For the log-linear specification, investment and $A_{t}$ are irrelevant to my research question.
} 
However, the tax that in a decentralized economy supports the equilibrium level of emissions is invariant to $v$. Let $X(n)$ denote aggregate equilibrium emissions, and denote $\tau=v^{\prime}(X(n) ; 1)$ as the tax that supports this level of emissions. If $v$ is the utility of consumption, then the tax equals marginal utility times the value of marginal product of emissions (units: utility/emissions). If utility is linear in consumption, so that $v$ is output as a function of emissions, then the tax equals the value of marginal product of emissions (units: money/emissions).

Proposition 5 For the linear-in-state model, the tax $\tau=v^{\prime}(X(n) ; 1)$ that supports the state-independent equilibrium is invariant to $v$ :

$$
\tau=-\frac{\kappa}{n}\left(i_{1}^{\prime}-(r-\lambda) \tilde{q}^{\prime}\right)(B-\lambda I)^{-1} C .
$$

The absolute value of the elasticity of this tax with respect to $n$ is 1 , and the elasticity of the tax with respect to $\lambda$ is

$$
\varepsilon=-\frac{d \tau}{d \lambda} \frac{\lambda}{\tau}=-\lambda \frac{\left[\tilde{q}^{\prime}+\left(i_{1}^{\prime}-(r-\lambda) \tilde{q}^{\prime}\right)(B-\lambda I)^{-1}\right](B-\lambda I)^{-1} C}{\left(i_{1}^{\prime}-(r-\lambda) \tilde{q}^{\prime}\right)(B-\lambda I)^{-1} C} .
$$

Each of the tribes takes into account only the climate-related damages that they suffer, $1 / n$ 'th of total damages. Therefore, the tribe's equilibrium tax is $1 / n$ 'th the tax chosen by a global planner with the same level of altruism.

The tax and elasticity formulae simplify if $S$ is a scalar. Denote $\hat{c}, \hat{B}$, and $\hat{C}=1$ as the scalar coefficients in the equation of motion. Here, $\Lambda=\hat{B}<0$ and $\tilde{q}=\frac{1}{\gamma-\hat{B}}$.

Corollary 3 For the case where $S$ is a scalar,

$$
\tau=\frac{\kappa(\theta-\hat{B}+\lambda)}{n(\lambda-\hat{B})(\gamma-\hat{B})}, \text { and } \varepsilon=\frac{\lambda \theta}{(\lambda-\hat{B})(\theta+\lambda-\hat{B})}
$$

$\varepsilon$ is independent of $r$ and increases with $\hat{B}$, reaching its upper bound at $\hat{B}=0$, where $\varepsilon=\frac{\theta}{\theta+\lambda}<1$ : as the pollutant becomes more persistent ( $\hat{B}$ increases toward 0$)$, the tax becomes more sensitive to $\lambda$. 
The elasticity $\varepsilon$ is maximized at $\lambda=\sqrt{\hat{B}^{2}-\hat{B} \theta}$. A $2 \% /$ year mortality implies $\varepsilon<0.33$ for a 100 year half-life of the stock, and $\varepsilon<0.51$ for a 300 year half-life.

In the scalar model, the effect of emissions on damages is immediate, and decays at a constant rate. Delayed and persistent effects of emissions require a higher dimensional state variable. The following corollary states that (particular) limiting values of the tax in the higher dimensional and the scalar models are equal. I denote $\Lambda^{\min }=\min _{i} \Lambda_{i}$ and $\Lambda^{\max }=\max _{i} \Lambda_{i}$, the minimum and maximum eigenvalues of $B$.

Corollary 4 (i) Where $S$ is a scalar, as $\hat{B}$ varies over its domain $(-\infty, 0)$, the constant tax varies monotonically over its range, $\left(0, \frac{\kappa(\theta+\lambda)}{n(r+\theta) \lambda}\right)$. (ii) Where $S$ is a vector, as all stock variables decay infinitely rapidly, the tax approaches 0 , and as all stocks become infinitely persistent, the tax approaches the upper bound of the scalar model. Formally: the constant tax approaches 0 as $\Lambda^{\max } \rightarrow-\infty$ and the constant tax approaches $\frac{\kappa(\theta+\lambda)}{n(r+\theta) \lambda}$ as $\Lambda^{\mathrm{min}} \rightarrow 0$ (from below).

Remark 1 Corollary 4.(ii) does not establish monotonicity: for some matrices B, the tax (and its elasticity) in the higher dimensional model might lie outside the range in the scalar model. To explore this possibility, I convert Gerlagh and Liski's (2012)'s discrete time climate model to my continuous time setting, and compute $\varepsilon$ using equation (11). For small values of $\lambda, \varepsilon<1$ (as in the scalar case). For sufficiently large $\lambda, \varepsilon>1$. This example shows the importance of non-monotonic dynamics, and illustrates the flexibility of the tools developed here.

The linear-in-state model makes the source of multiplicity and dominance transparent. If all social planners believe that other tribes and their own successors use state-independent policies, the auxiliary optimal control problem used to obtain the equilibrium is linear in the state, and the shadow value of the state variable $\left(g^{\prime}\right)$ does not depend on other agents' actions. The solution to that problem is a state-independent emissions policy that is independent of the other agents' strategies. Thus, the equilibrium strategy is dominant and unique, within the class of state-independent policies. If, instead, the planner believes that the other agents' policy rule is a non-linear function of the state, then the planner's auxiliary optimal 
control problem is non-linear in $S$. In this case, the planner's optimal policy is state-dependent, and in general not a dominant strategy.

Iverson (2013) shows, for the discrete-time log-linear model with investment and $n=1$, that the first period action of a planner who can commit does not depend on her beliefs about future state-independent actions; this first period action equals the constant policy in a state-independent MPE. Phelps and Pollack (1968) obtain this result for a simpler model. This result is consistent with the dominance result in Proposition 4.ii, which holds for arbitrary $n$ and concave function $v$.

The linear-in-state model with state-independent policies is a good place to begin, but these functional assumptions imply that the unique equilibrium is in dominant strategies. The functional assumptions therefore eliminate the strategic interactions both across countries and generations. These issues are central to climate policy, and the focus of this research. In addition, the model implies that the elasticity of the equilibrium with respect to either international cooperation $(n)$ or intergenerational altruism $(\lambda)$ does not depend the other variable.

\subsection{The linear-quadratic model}

To overcome these limitations, I assume that damages are quadratic rather than linear in the state. Here, an agent's shadow value of the state depends on the state, and thus depends on other agents' actions: the strategic interactions across tribes and generations are non-trivial.

I also broaden the research question to study the multiplicity of equilibria. For the linear-in-state model, the state-independent equilibrium is the limit equilibrium to a finite horizon model as the horizon approaches infinity. For the linear-quadratic model in this section, the linear equilibrium is the limit equilibrium. There are many other equilibria, but even numerical computation of those is an unsolved problem unless $S$ is a scalar. ${ }^{8}$ I therefore consider a scalar model, eliminating non-monotonic

\footnotetext{
${ }^{8}$ Numerical methods using function iteration can find a differentiable MPE for a general (nonscalar) model. However, experience with these methods suggests that they identify a single equilibrium, the limit of the equilibrium to a finite horizon model (Fujii and Karp (2008)). I have not succeeded in using these methods to study the kind of multiplicity of MPE discussed above.
} 
impulse-response functions. Remark 1 illustrates that ignoring those features of the climate problem can impact model results. The choice is between studying multiplicity of equilibria with simple dynamics, or a single equilibrium with rich dynamics. I choose the former, leaving the latter to work in progress. Appendix $\mathrm{C}$ contains supplementary material for this section.

\subsubsection{Calibration}

The scalar state variable, $S$, is the atmospheric stock of carbon, in parts per million (ppm) by volume. The control variable (previously $x$ ) is abatement, denoted $A$, defined as the percent reduction in Business as Usual (BAU) emissions. The flow payoff is a quadratic function of the stock and abatement, and the equation of motion is linear in the stock and abatement.

The time derivative of the stock of atmospheric carbon, the function $f\left(S_{t}, A_{t} ; 1\right)$, is linear in $S, A$. This function contains three parameters: an intercept and the coefficients of $S$ and $A$. I use the following three calibration assumptions:

(i) The steady state absent anthropogenic emissions is $280 \mathrm{ppm}$.

(ii) The half-life of the atmospheric stock is 83 years.

(iii) The year 2100 BAU stock is 700 ppm, given 2010 stock of 380 .

The first calibration assumption is non-controversial. The 83-year half life is slightly less than the level used in DICE (Nordhaus 2008). The IPPC's 2007 projections of year 2100 stocks range from 535 to 983 ppm. My definition of BAU means literally incurring no cost to reduce emissions, whereas the IPPC's projections refer to levels that are likely to occur. The IPPC lower bound of $535 \mathrm{ppm}$ represents a $24 \%$ reduction relative to my assumed BAU. My assumptions imply that under BAU, the stock reaches 870 ppm after 200 years.

For the aggregate economy $(n=1)$, the flow payoff is $-\frac{1}{2}\left(b A^{2}+h(S-280)^{2}\right)=$ $-\frac{1}{2} b\left(A^{2}+\frac{h}{b}(S-280)^{2}\right)$. The flow cost is the sum of a term proportional to the square of abatement and to the square of the increase in the stock relative to preindustrial levels, so damages are convex. To calibrate the model, I define $\Omega \equiv \frac{h(560-280)^{2}}{b 50^{2}}$, a 
parameter that equals the flow cost of doubling the stock of GHG, relative to preindustrial levels, as a ratio of the flow cost of a $50 \%$ reduction in BAU emissions. In view of the amount of uncertainty about both abatement costs and climate damages, I calibrate the model using the ratio of these, instead of the coefficients $h$ and $b$ separately. This approach makes it easy to consider a range of beliefs about relative damages and costs, without the need to consider independently damages and costs. The model solves for equilibrium $A$ as a function of the stock $S$.

Karp and Zhang (2006) estimate an abatement cost parameter that matches (with $R^{2}=0.97$ in a psuedo-regression) the Nordhaus (1994) cost assumptions. ${ }^{9}$ That estimate implies that a 50\% abatement reduces Gross World Product (GWP) by 1.1\%. A low-to-moderate estimate of damages from doubling stocks is $1.33 \%$ of GWP. These estimates suggest that $\Omega=\frac{1.33}{1.12} \approx 1.2$ is consistent with (at least some) previous modeling efforts. I report results for $\Omega=1$ (low damages) and $\Omega=3$ (moderate damages). As a plausibility check for the range $\Omega \in[1,3]$, note that given the calibration assumptions for the equation of motion, $\Omega$ determines the UMSS (the Utility Maximizing Steady State). For $\Omega=1$, UMSS $=553 \mathrm{ppm}$ and for $\Omega=3$, UMSS $=402 \mathrm{ppm}$. A steady state carbon stock of $450 \mathrm{ppm}$ has been proposed as a reasonable target. This target incorporates some discounting, whereas the UMSS effectively sets the discount rate to zero. Thus, a UMSS of $402(\Omega=3)$ appears to be broadly consistent with the 450 ppm target; the UMSS of $553(\Omega=1)$ is consistent with the view that it is not worth doing much to slow emissions.

The model captures many features of the climate problem using four climaterelated parameters. To be useful, numerical results require that model inputs be transparent. The three parameters of particular interest are: (i) $\Omega$, a measure of the damages from increased carbon stock relative to the costs of abatement; (ii) $\lambda$, an inverse measure of intra-tribal altruism; (iii) $n$, an inverse measure international cooperation. I show above that $\Omega$ is consistent with "prevailing views".

The choice of $\lambda$ and $n$ presents different problems. These parameters have different units, and the elasticities of interest depend on the current stock. In

\footnotetext{
${ }^{9}$ Significantly, I use this early cost estimate only to obtain an idea of a reasonable magnitude of $\Omega$. Despite refinements in cost estimates, I do not think that they have changed by multiples.
} 
addition, I assume agents are symmetric, but in the real world, nations are not. An increase in $n$ represents less international cooperation, but what represents an empirically relevant level of $n$ ? Also, the range of parameters is large: $\lambda \in(0, \infty]$ and $n \in[1, \infty]$. These difficulties are easily overcome. With a unit of time of one year, I choose $r=0.02=\theta$, an annual pure rate of time preference and mortality of $2 \%$. I begin with the linear equilibrium, where the equilibria under $\lambda=0.1$ and $\lambda=\infty$ are similar; $\lambda=\infty$ implies zero altruism and $\lambda=0.1$ implies such a low level of altruism as to have only a small affect on the outcome. As $n \rightarrow \infty$ all international cooperation vanishes, and zero abatement is the only equilibrium. Abatement is low for $n=10$ : this value therefore implies a high degree of fragmentation. Thus, $\lambda \in[0,0.1]$ and $n \in[1,10]$ provide reasonable bounds for studying parameter changes.

\subsubsection{The linear equilibrium in the linear-quadratic model}

In the linear equilibrium I find: (i) The equilibrium is not sensitive to whether we use the exponentially distributed lifetime or the finite deterministic lifetime model. (ii) The degree of fragmentation $(n)$ has a much larger effect on the equilibrium outcome, compared to the degree of altruism $(\lambda)$; and (iii) for moderately high levels of fragmentation, the degree of altruism is unimportant.

Finding the linear equilibrium in the infinite horizon model requires finding a root of a cubic satisfying an inequality that is necessary and sufficient for the equilibrium state trajectory to converge, and thus satisfies a transversality condition. The coefficients of both the cubic and the inequality are complicated functions of the model parameters, excluding further analytic results. However, the numerical problem is trivial. For all the cases I consider, I find a unique linear equilibrium.

I report results for the year 2100 stock, a level often used in policy discussions, for $r=0.02=\theta=\frac{1}{T}$ (a risk-adjusted discount rate of $4 \%$ ), using $\lambda \in\{0,0.02,0.1, \infty\}$ to represent a range of altruism, $n \in\{1,5,10\}$ to represent a range of fragmentation amongst tribes, and $\Omega \in\{1,3\}$ to represent a range of beliefs about the cost of climate change relative to the cost of abatement. Table 2 shows the equilibrium reduction in year 2100 stock, as a percent of the BAU stock in that year (hereafter, 
"stock reduction"). The left panel corresponds to the agent with exponentially distributed lifetime, and the right panel corresponds to the agent with finite lifetime, both with paternalistic altruism. The first element in an entry corresponds to low damages, $\Omega=1$, and the second element corresponds to moderate damages, $\Omega=3$. Keeping the year 2100 stock to 400, 500 and 600 ppm requires, respectively, stock reductions of $43 \%, 29 \%$ and $14 \%$ of the BAU levels of $700 \mathrm{ppm}$. For the parameter values here, the equilibrium year 2100 stock ranges from $470 \mathrm{ppm}$ (with $n=1$ and $\lambda=0$ ) to just under $700 \mathrm{ppm}$ for large values of $n$ and $\lambda$.

\begin{tabular}{|c|c|c|c|}
\hline$\lambda \backslash n$ & 1 & 5 & 10 \\
\hline 0.0 & $(19,33)$ & $(6,12)$ & $(3,8)$ \\
\hline 0.02 & $(11,25)$ & $\overline{(2,7)}$ & $\overline{(1,4)}$ \\
\hline 0.1 & $(8,1 !$ & $(2,5$ & $(1,2)$ \\
\hline$\infty$ & $(7,17)$ & $(2,4)$ & $(1,2)$ \\
\hline
\end{tabular}

\begin{tabular}{|c|c|c|c|}
\hline$\lambda \backslash n$ & 1 & 5 & 10 \\
\hline 0.0 & $(24,37)$ & $(7,15)$ & $(4,10)$ \\
\hline 0.02 & $(11,25)$ & $(2,7)$ & $(1,4)$ \\
\hline 0.1 & $(5,14)$ & $(1,3)$ & $(0.5,2)$ \\
\hline$\infty$ & $(3,9)$ & $(0.7,2)$ & $(0.3,1)$ \\
\hline
\end{tabular}

Table 2: Reduction in the year 2100 stock as a \% of BAU level ("stock reduction"). Rows vary the altruism parameter, $\lambda$, and columns vary the fragmentation parameter, $n$. First element corresponds to low relative damages, $\Omega=1$, and second corresponds to moderate relative damages, $\Omega=3$. Table 2.I corresponds to the agent with exponentially distributed lifetime and Table 2.II corresponds to the agent with finite lifetime.Table 2 justifies my emphasis on parameter ranges $\lambda \in[0,0.1]$ and $n \in[1,10]$.Equilibrium abatement under $\lambda=0.1(=5 \times r)$ is similar to equilibrium abatement under $\lambda=\infty$. Equilibrium abatement for $n=10$ is small, and thus similar to equilibrium abatement under $n=\infty$. The rows corresponding to $\lambda=0.02=r$ in the two panels are identical, because for $\lambda=r$ the two problems are equivalent; both have the constant discount rate $r+\theta=r+\frac{1}{T}$. Comparisons between other rows illustrate the points made in Section 2.3. Figure 1 shows that for $t>0$, the discount rate corresponding to exponentially distributed lifetime is less than the discount rate corresponding to finite deterministic lifetime if $\lambda>r$; the relation between discount rates is reversed if $\lambda<r$. The two panels in Table 2 reflect these relations: for $\lambda>r$, equilibrium abatement is higher under the exponential than the deterministic case; $\lambda<r$ reverses the comparison. The absolute difference between discount rates is greatest for large 
$\lambda>r$. Reflecting this fact, the ratio of equilibrium abatement levels (expressed as a number greater than 1), under the two assumptions about lifetime, is greatest for $\lambda=\infty$. However, because the equilibrium levels are similar under $\lambda=0.1$ and $\lambda=\infty$, the difference in the ratios is likely not of great practical significance. My assessment is that for this model, the difference between the exponentially distributed and finite deterministic model is of second order importance. That conclusion might not hold under different calibrations; thus, it is important to have the formulae. The rest of this paper discusses only the agent with exponentially distributed lifetime.

An increase of $n$ from 1 to 5 causes a much larger equilibrium change, relative to an increase in $\lambda$ from 0 to 0.02 . Changing both parameters from their lower bound to the moderate levels causes, for $\Omega=3$, a nearly $80 \%$ fall in equilibrium stock reduction; an increase in only $\lambda$ causes a $24 \%$ fall in stock reduction and an increase in only $n$ causes a $64 \%$ fall in stock reduction. By these measures, a decrease in contemporaneous cooperation has a much larger effect on the equilibrium than does a decrease in altruism. This conclusion echoes Corollary 3.

Increasing $\Omega$ from 1 to 3 , holding $n$ and $\lambda$ fixed, increases the stock reduction by a factor of $2-3$ in most cases. An increase in $n$ from 1 to 5 leads to a much larger stock reduction, in both percentage and absolute terms, than the reduction caused by a change of $n$ from 5 to 10 . Cooperation breaks down rapidly even at small levels of fragmentation. A larger value of $n$ decreases the effect of changes in altruism. If there is little contemporaneous cooperation, the degree of altruism is rather unimportant. For example, at $n=10$ equilibrium stock is close to BAU levels for all values of $\lambda$, so changes in $\lambda$ do not have much effect. In contrast, even at high values of $\lambda$, a change in $n$ has a large effect on the equilibrium.

For $\Omega=3, n=1$ and $\lambda=0$, the equilibrium steady state is $496 \mathrm{ppm}$, an almost $50 \%$ reduction in the BAU steady state. The same steady state results from a standard optimization problem with a constant discount rate of $0.9 \%$. As noted above, the stock that maximizes the steady state utility flow (the "UMSS") is 402 ppm; the same steady state results from the social planner's problem in the limit as the constant discount rate approaches 0 . 


\subsubsection{Nonlinear equilibria in the linear-quadratic model}

For non-linear equilibria and the agent with exponentially distributed lifetime: (i) Altruism and fragmentation are similarly important in determining equilibrium behavior; and (ii) Altruism may be especially important in very fragmented environments. Thus, consideration of non-linear equilibria can reverse the conclusions obtained under the assumption of a linear equilibrium. I establish these claims by first comparing equilibrium policy rules, and then examining the effect of $\lambda$ and $n$ on a particular steady state. Non-linear equilibria - unlike the linear equilibrium - may be defined over only a strict subset of state space. The three-dimensional system $(S, A, K)$ does not permit a graphical analysis of the domain of existence of non-linear equilibria. However, where $S$ is a scalar, nonlinear equilibria can be obtained by solving the system of differential equations for $A$ and $K$ as functions of $S$, as an initial value problem. The domain of existence of (many) nonlinear equilibrium policy functions is large, so these equilibria are not vacuous.

Figure 2 illustrates the multiplicity of equilibria for $n=5, \Omega=3, \lambda=0.00001$, and the other parameters given above. The dashed line shows the graph of $f(S, A ; 1)=$ 0 , the set of steady states, between the initial stock, 380, and the steady state in the linear equilibrium, 760. At points $(S, A)$ strictly below this line, the stock is increasing over time. Each solid graph shows a particular equilibrium decision rule, giving abatement $(A)$ as a function of the stock $(S)$. The numerical label on each of these three graphs shows the steady state induced by that policy function. The positively sloped line is the linear equilibrium, with a steady state of $760 \mathrm{ppm}$. In this equilibrium, the level of abatement increases with the stock. The non-monotonic policy function drives the state to $745 \mathrm{ppm}$. In this equilibrium, abatement starts out high and falls until the stock gets close to its steady state; in the neighborhood of the steady state, abatement increases. Even though the two steady states, 760 and 745 , are similar, the speed of increase in much higher in the linear equilibrium. As a consequence of the scale of the figure, the curve labeled "463 ppm" appears to be coincident with the dashed line. The actual curve lies below the dashed line except at the steady state, so this policy rule drives any stock $380 \leq S_{0} \leq 463$ to the 


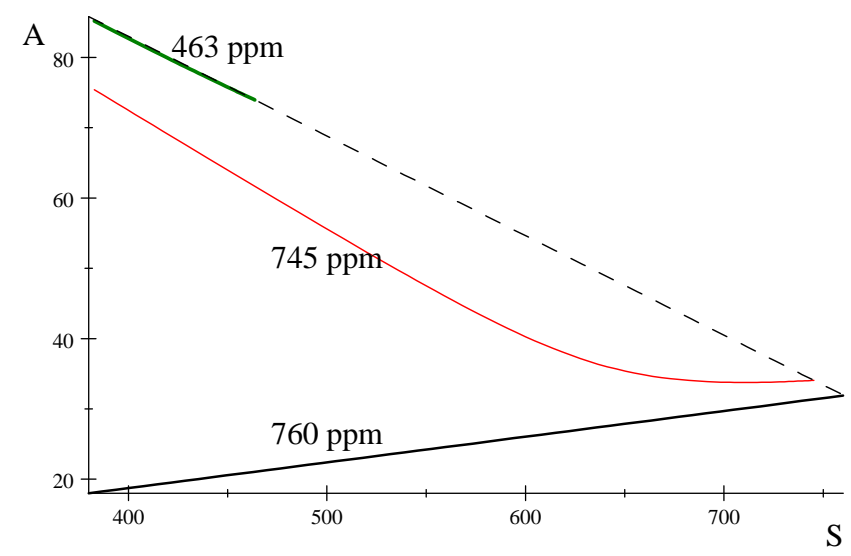

Figure 2: Abatement, $A$ (as a percent of BAU emissions) as a function of the stock, $S$ (ppm), for $n=5, \lambda=0.00001$ and $\Omega=3$. The three MPE policy functions drive the state from the initial value $380 \mathrm{ppm}$ to different steady states, shown by the labels on the curves. The dashed line shows the combinations of abatement and stock that maintain a steady state.

steady state 463 . In this equilibrium, abatement falls over time, allowing the stock to grow slowly until it reaches the steady state. The stock 463 is slightly above the commonly used target of $450 \mathrm{ppm}$, and is about 10\% larger than the infimum steady state (discussed below) with $n=5, \Omega=3$, and $\lambda=0.00001$.

Figure 2 has two messages. First, the domain of existence of non-linear equilibria that maintain low stock levels may include the current stock level: the multiplicity of equilibria is not a mere curiosity. With a moderate to high degree of fragmentation $(n=5)$ and a high degree of altruism $(\lambda=0.00001)$, equilibrium abatement might result in either a very high or moderately low stock. Second, actions may be strategic complements or substitutes, or may switch from one to the other as the state variable changes. Where the equilibrium decision rule is increasing in the stock, actions are strategic substitutes: a higher level of abatement by an agent today leads to a lower future stock, with lower future equilibrium abatement by all agents. Conversely, when the decision rule is decreasing in the stock, actions are strategic complements: an agent has an incentive to abate at a high current level in order to keep the 
stock from growing, and thus maintaining high future abatement. Consequently, strategic complementarity is associated with relatively high levels of abatement, slow increases in the stock, and low steady state stocks. Strategic complementarity implies especially large abatement efforts early in the trajectory, while the stock is still low. ${ }^{10}$ In contrast, if actions are strategic substitutes, an agent has an incentive to abate at a low level, knowing that the higher future stock will increase future abatement. Thus, strategic substitutes correspond to low abatement, rapid growth in the stock, and a high steady state stock.

The set of steady states that can be supported as a MPE is an open interval. I define $\beta$ as the infimum of this set, as a ratio of the UMSS; $\beta$ is a function of $\lambda$ and $n$ and other model parameters, and provides a measure of the distance between the UMSS and the set of steady states. Proposition 3 implies that for $n=1, \beta \rightarrow 1$ as $\lambda \rightarrow 0$. The solid graphs in Figure 3 show $\beta$ for $0 \leq \lambda \leq r=0.02$ for $n=1$ and $n=5$, given moderate damages $(\Omega=3)$ and the other parameter values discussed above. The dashed graphs show, for different values of $\lambda$ and $n$, the steady state in the linear equilibrium as a ratio of UMSS.

For $\lambda=r$ and $n=1$ the game is a standard optimal control problem, so the linear equilibrium is the unique MPE; consequently, for $n=1$ the solid and dashed graphs converge at $\lambda=r$. For $\lambda<r$ or $n>1$ there are multiple equilibria. In this circumstance, the gap between the smallest stable MPE steady state and the steady state under the linear equilibrium (reflected in the distance between a solid and a dashed curve) increases with both $n$ and $r-\lambda$. Higher $n$ or $r-\lambda$ increase the coordination problem arising from the multiplicity of equilibria.

For small values of $\lambda$ it is possible to support, as a MPE steady state, a GHG stock close to UMSS regardless of whether $n=1$ or $n=5$. (For small $\lambda$, the solid curves corresponding to $n=1$ and $n=5$ are close to each other and both close to 1.) However, for larger values of $\lambda$, the smallest possible steady state in a MPE is

\footnotetext{
${ }^{10}$ The possibility that equilibrium abatement falls over time, ignores the likely reality of convex adjustment costs in emissions levels. It also ignores that abatement costs may fall over time, an assumption that is important to the "policy ramp" in DICE, but absent in my stationary model. These simplifications, and many others, mean that this model is not suitable for detailed policy advice; as noted above, it does not have that normative objective.
} 


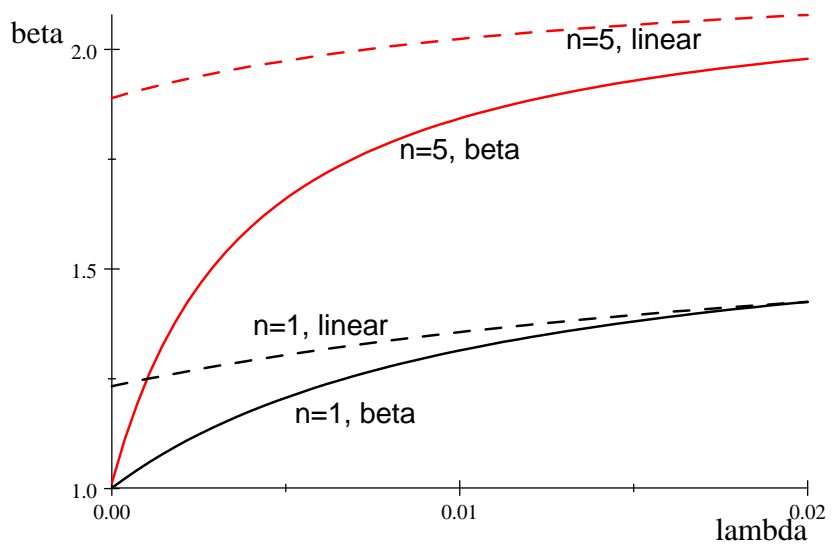

Figure 3: Solid curves show $\beta$, the ratio of the infimum of stable MPE steady state to the UMSS. Dashed curves show ratio of steady state in linear equilibrium to the UMSS. Moderate damages, $\Omega=3$.

much larger for $n=5$ compared to $n=1$. Thus, with non-linear equilibria, the altruism parameter becomes more important for larger $n$. Section 4.2 .2 notes that with linear equilibria, the altruism parameter is less important for large $n$.

\section{Discussion}

The provision of a long-lived public good depends on the ability of contemporaneous agents to cooperate, and on their degree of altruism towards future generations. Limited altruism and lack of cooperation are both first order obstacles to effective climate policy. Which is a greater obstacle, and how do the two interact? There can be no general answer to such a question. Any answer is conditional on assumptions about the model and the equilibrium set. This paper sheds light on the question by providing a framework for addressing it. It applies that framework to obtain analytic answers in a model with linear climate-related damages, and numerical answers in a model with convex damages. In constructing the framework, I obtain results of independent interest, clarifying the relation between the induced aggregate discount factors for agents with paternalistic or pure altruism, and with exponentially 
distributed or finite deterministic lifetimes.

For the scalar linear-in-state model, I show analytically that the elasticity of the constant equilibrium tax with respect to the measure of fragmentation exceeds (easily by a factor of 2 - 3) the magnitude of the elasticity with respect to the degree of altruism: here, lack of international cooperation is a much bigger impediment to climate policy than is limited altruism toward future generations. The linear-in-state model is valuable for intuition, but regardless of its dimension it obscures inter- and intra-generational strategic interactions: the equilibrium state-independent policies are dominant, i.e. they do not depend on the actions that other agents take.

I therefore consider a linear quadratic model, where damages are convex in the pollution stock. Here, there are non-trivial inter- and intra-generational strategic interactions in all equilibria. I find that the relative importance of the two obstacles depends on the equilibrium type. The linear equilibrium has a special claim to our attention; it is the limit of the unique equilibrium to a finite horizon game, and where it exists it is defined over the entire state space. If one considers only the linear equilibrium in the climate application, the two conclusions for the scalar linear quadratic model are: (i) the degree of contemporaneous cooperation has significantly greater effect on equilibrium policy than does the degree of intergenerational altruism, and (ii) once the degree of cooperation is even moderately low, the outcome is insensitive to the degree of altruism. Conclusion (i) echoes the comparison of elasticities for the constant policies in the linear-in-state model.

However, non-linear equilibria, which owe their existence to the infinite horizon, can support a broad range of outcomes, some with high levels of abatement and low pollution stocks. Here, the outcome may be as or even more sensitive to the degree of altruism than to the level of cooperation, and the degree of altruism may be important especially when the degree of cooperation is low. The domain of non-linear equilibria can be large enough to be relevant.

This summary pertains to the scalar model, which cannot capture delayed and persistent effects of emissions on damages. A higher dimensional model can capture those effects, but with (my knowledge of) available methods, that model cannot be used to study the multiplicity of equilibria. Investigation of a particular equilibrium 
for the higher dimensional model is the subject of current research.

A general conclusion is that there may be many equilibria in games that determine the provision of a long-lived public good by countries that care about only their own current and future citizens. The logic of Nash's noncooperative equilibrium does not doom us to bad outcomes, even if we exclude trigger or other punishment strategies. (Consideration of such strategies increases the equilibrium set, leading to the possibility of still better outcomes.) This conclusion, although not specific to this paper, is nevertheless worth stating, because many non-cooperative models of climate policy build in strategic substitutability, implying that agents have an incentive to undertake less public investment, in order to increase future public investment. This built-in free riding causes the models to be quite pessimistic about the chance of a meaningful climate agreement amongst sovereign nations. Recognition of the possibility of strategic complementarity, where agents have an incentive to increase their current investment in order to increase future investment, moderates this pessimism. International negotiations on climate policy are important, even if they do not result in enforceable agreements. Negotiations make coordination on a good equilibrium easier to achieve. 


\section{References}

Aldy, J., And R. Stavins (eds.) (2007): Architectures for Agreement: Addressing Global Climate change in the Post-Kyoto World. Cambridge Univerisity Press.

Andreoni, J. (1989): "Giving with impure altruism: applications to charity and Ricardian equivalence," Journal of Political Economy, 97, 1447-1458.

BArro, R. (1999): "Ramsey meets Laibson in the neoclassical growth model," Quarterly Journal of Economics, 114, 1125-52.

Blanchard, O. J. (1985): "Debts, deficits and finite horizons," Journal of Political Economy, 93, 223-247.

Diamond, P. A. (1965): "National Debt in a Neoclassical Growth Model," The American Economic Review, 55(5), 1126-1150.

Dockner, E., And N. van Long (1993): "International pollution control: cooperative versus non-cooperative strategies," Journal of Environmental Economics and Management, 24, 13-29.

Dutta, P. K., and R.-K. Sundaram (1993): "How Different Can Strategic Models Be?," Journal of Economic Theory, 60, 42-61.

Ekeland, I., L. Karp, and R. Sumaila (2012): "Equilibrium management of fisheries with altruistic overlapping generations," University of British Columbia Working Paper.

Ekeland, I., AND A. LAzRAK (2010): "The golden rule when preferences are time inconsistent," Mathematical and Financial Economics, 4(1), 29-55.

Fershtman, C., and M. Kamien (1987): "Dynamic Duopolistic Competition with Sticky Prices," Econometrica, 55, 1151-1164.

FudenberG, D., And D. Levine (1983): "Subgame perfect equilibria of finite and infinite horizon games," Journal of Economic Theory, 31, 251-268. 
FuJII, T., AND L. KARP (2008): "Numerical analysis of non-constant pure rate of time preference: a model of climate policy," Journal of Environmental Economics and Management, forthcoming, 56, 83-101.

Gerlagh, R., And M. Liski (2012): "Carbon prices for the next thousand years," Unpublished Working Paper.

Gollier, C., And M. Weitzman (2010): "How should the distant future be discounted when discount rates are uncertain?," Economic Letters, 107, 350-353.

Golosov, M., J. Hassler, P. Krusell, and A. Tsyvinski (2011): "Optimal taxes on fossil fuels in general equilibrium," NBER Working Paper 1738.

Guersnerie, R. (2004): "Calcul economique et developpement durable," Revue economique, 55(3), 363-382.

Guesnerie, R., And H. Tulkens (eds.) (2008): The Design of Climate Policy. MIT Press.

Harris, C., And D. Laibson (2001): “Dynamic Choices of Hyperbolic Consumers," Econometrica, 69(5), 935-957.

Haurie, A., J. Krawczyk, And G. Zaccour (2012): Games and Dynamic Games. Imperial College Press; World Scientific, Hackensack, New Jersey.

Hoel, M., And T. Sterner (2007): "Discounting and Relative Prices," Climatic Change, 84, 265-80.

IVERson, T. (2013): "Optimal carbon taxes with non-constant time preference," http://mpra.ub.uni-muenchen.de/43264/.

JACKSON, M., AND L. YARIV (2011): "Collective dynamic choice: the necessity of time inconsistency," Department of Economics, Stanford, Working Paper.

KARP, L. (2005): "Global Warming and hyperbolic discounting," Journal of Public Economics, 89, 261-282. 
- (2007): "Non-constant discounting in continuous time," Journal of Economic Theory, 132, $557-568$.

Karp, L., and J. Zhang (2006): "Regulation with Anticipated Learning about Environmental Damage," Journal of Environmental Economics and Management, 51, 259-280.

Krusell, P., and A. Smith (2003): "Consumption-saving desisions with quasigeometric discounting," Econometrica, 71(1), 365-75.

Kubiszewski, I., R. Costanza, C. Franco, P. Lawn, J. Talbreth, T. JackSon, and C. Aylmer (2013): "Beyond GDP: Measuring and achieving global genuine progress," Ecological Economics, 93, 57-68.

LaIBSOn, D. (1997): "Golden eggs and hyperbolic discounting," Quarterly Journal of Economics, 62, 443-78.

Levhari, D., and L. Mirman (1980): "The Great Fish War: An Example Using a Dynamic Cournot-Nash Solution," Bell Journal of Economics, 11, 322-334.

Li, C. Z., And K. G. Lofgren (2000): "Renewable Resources and Economic Sustainability: A Dynamic Analysis with Heterogeneous Time Preferences," Journal of Environmental Economics and Management, 40, 236-250.

Long, N. V. (2010): A Survey of Dynamic Games in Economics. World Scientific, Singapore.

Nordhaus, W. (1994): Managing the Global Commons: The economics of the greenhouse effect. Cambridge, MA: MIT Press.

Nordhaus, W. D. (2007): "A Review of the Stern Review on the Economics of Climate Change," Journal of Economic Literature, 45(3), 686 - 702.

Nordhaus, W. D. (2008): A Question of Balance. Yale University Press. 
Phelps, E., And R. Pollack (1968): "On Second-best National Savings and Game: Equilibrium Growth," Review of Economic Studies, 35, 185-199.

Ramsey, F. (1928): "A Mathematical Theory of Savings," Economic Journal, 38, $543-59$.

RAY, D. (1987): "Nonpaternalistic intergenerational altruism," Journal of Economic Theory, (41), 112-132.

Reynolds, S. (1987): "Capacity Investment, Preemption and C ommitment," International Economic Review, 28, 69-88.

Saez-Marti, M., and J. Weibull (2005): "Discounting and altruism to future decision- makers," Journal of Economic Theory, (122), 254-66.

Schneider, M., C. Traeger, and R. Winkler (2012): "Trading off generations: equity, efficiency and climate change," forthcoming, European Economic Review.

Stern, N. (2007): The Economics of Climate Change. Cambridge University Press.

Strotz, R. (1956): "Myopia and Inconsistency in Dynamic Utility Maximization," Review of Economic Studies, 23, 165-180.

Sumaila, U., and C. Walters (2005): "Intergenerational discounting: a new intuitive approach," Ecological Economics, 52, 135-142.

Traeger, C. (2012a): "Sustainabililty, limited substitutability and non-constant social discount rates," Journal of Environmental Economics and Management, 62, $215-228$.

— (2012b): "Why uncertainty matters - Discounting under Intertemporal risk aversion and ambiguity," DARE Working Paper 1092R2.

Tsutsui, S., And K. Mino (1990): "Nonlinear Strategies in Dynamic Duopolisitc Competition with Sticky Prices," Journal of Economic Theory, 52, 136-161. 
VAn der Ploeg, R., And A. De Zeeuw (1992): "International aspects of pollution control," Environmental and Resource Economics, 2, 117-39.

Vieille, N., AND J. WeiBull (2009): "Multiple solutions under quasi-exponential discounting," Economic Theory, 39, 513-26.

Weitzman, M. (2007): "A Review of the Stern Review on the Economics of Climate Change," Journal of Economic Literature, 45(3), 703-724.

WirL, F. (1994): "Pigouvian taxation of energy for stock and flow externalities," Environmental and Resource Economics, 26, 1-18.

YAARI, M. E. (1965): "Uncertain lifetime, life insurance and the Theory of the Consumer," Review of Economic Studies, 32, 137-150.

Zuber, S. (2010): "The aggregation of preferences: can we ignore the past?," Theory and Decision, 70, $367-384$. 


\section{Referees' Appendices: not for publication}

The following three appendices provide an exhaustive compilation of calculations, proofs, and discussion of the model, presented in a manner to make it relatively easy for a referee to check any detail. I expect that little if any of this material needs to be published, although sketches of the proofs of Lemma 1, Propositions 1, 3, and 5, and Corollaries 3 and 4 should be made available in an online appendix.

I re-purpose some symbols, in order to avoid using obscure symbols. For example, in Section 4.1, $\varepsilon$ is an elasticity, whereas in Appendix A, $\varepsilon$ is the length of a period in a discrete time setting. Because the different appendices and the text are selfcontained, I expect that this practice will not create confusion.

\section{A Discounting}

This appendix contains the proofs of Propositions 1 and 2. Ekeland and Lazrak (2010) provide the continuous time discount factor under exponentially distributed lifetime and paternalistic altruism, equation (1), without the discrete time detour; I include that case so that this appendix is self-contained. I begin with a discrete time model, in which each period lasts for $\varepsilon$ units of time (e.g., years), and obtain the discount factors in the continuous time setting by passing to the limit, as $\varepsilon \rightarrow 0$. This approach is useful for explaining the meaning of the different models, and particularly for deriving the discount functions under pure altruism and for a finite lifetime.

\section{A.1 The discrete time model}

Table 3 introduces notation used to obtain concise expressions of the discrete time discount factors. The pure rate of time preference that an agent uses to evaluate her selfish component of welfare is $r$, and $\lambda$ is the rate she uses to evaluate the utility or welfare of future generations; $\rho=e^{-r \varepsilon}$ and $\delta=e^{-\lambda \varepsilon}$ are the corresponding discount factors. For the case of exponentially distributed lifetime, $\theta$ is the mortality $=$ birth $=$ hazard rate, so $\gamma=r+\theta$ is the risk-adjusted discount rate and $\alpha=e^{-\gamma \varepsilon}$ is the 
corresponding risk-adjusted discount factor. With a constant population normalized to $1, b=1-e^{-\theta \varepsilon}$ is the mass of agents born at the end of a period of length $\varepsilon$. In order to make the models with exponentially distributed and deterministic lifetimes comparable, I assume throughout that the lifetime in the latter case equals $T=\frac{1}{\theta}$, the expected lifetime under the exponential distribution.

\begin{tabular}{|l|l|l|l|l|}
\hline & mortality & $\begin{array}{c}\text { selfish time } \\
\text { preference }\end{array}$ & $\begin{array}{c}\text { risk adjusted } \\
\text { discounting }\end{array}$ & $\begin{array}{c}\text { altruism } \\
\text { weight }\end{array}$ \\
\hline $\begin{array}{c}\text { continuous } \\
\text { time rates }\end{array}$ & $\theta=\frac{1}{T}$ & $r$ & $\gamma=r+\theta$ & $\lambda$ \\
\hline $\begin{array}{c}\text { discrete time } \\
\text { factors }\end{array}$ & $b=1-e^{-\theta \varepsilon}$ & $\rho=e^{-r \varepsilon}$ & $\alpha=e^{-\gamma \varepsilon}$ & $\delta=e^{-\lambda \varepsilon}$ \\
\hline
\end{tabular}

Table 3: Parameters that appear in the discount functions

\section{A.1.1 Exponentially distributed lifetime, paternalistic altruism}

Agents use the risk-adjusted discount factor $e^{-\gamma \varepsilon}$ to evaluate their own future utility. Agents alive in a period are identical, so any can be chosen as the social planner who makes the decision about investment in the public good in that period. The social planner alive in period 0 gives weight $e^{-\gamma \varepsilon t}$ to the period $t$ utility of agents currently alive, and the weight $\left(1-e^{-\theta \varepsilon}\right) e^{-\lambda \varepsilon \tau} e^{-\gamma \varepsilon(t-\tau)}$ to the period $t$ utility of agents born in period $\tau \leq t$. There are $1-e^{-\theta \varepsilon}$ of these agents, each of whom discounts her period $t$ utility at $e^{-\gamma \varepsilon(t-\tau)}$, and the current social planner values that utility at $e^{-\lambda \varepsilon \tau}$.

The total weight that the current social planner puts on period $t$ utility flow (the discount factor) is the sum of the selfish and altruistic components for those currently alive:

$$
D(t ; \varepsilon)=e^{-\gamma \varepsilon t}+\left(1-e^{-\theta \varepsilon}\right) \sum_{\tau=1}^{t} e^{-\lambda \varepsilon \tau} e^{-\gamma \varepsilon(t-\tau)} .
$$




\section{A.1.2 Exponentially distributed lifetime, pure altruism}

The expected present value of the flow of utility of an agent alive in period $\tau$ (the selfish component of her welfare) is

$$
\sum_{t=0}^{\infty} e^{-\gamma \varepsilon t} u_{\tau+t}
$$

An agent's total welfare equals the sum of her own selfish utility and the utility that she receives from the welfare of agents who are born in the future. Denote the total welfare of the agent born in period $\tau+t$ as $V_{\tau+t}$. In each period, $1-e^{-\theta \varepsilon}$ agents are born. The agent currently alive attaches the weight $\left(1-e^{-\theta \varepsilon}\right) e^{-\lambda \varepsilon t}$ to the welfare of the generation born $t$ periods in the future. Thus, the welfare of the agent alive at period $\tau$ is ${ }^{11}$

$$
V_{\tau}=\sum_{t=0}^{\infty} e^{-\gamma \varepsilon t} u_{\tau+t}+\left(1-e^{-\theta \varepsilon}\right) \sum_{t=1}^{\infty} e^{-\lambda \varepsilon t} V_{\tau+t}
$$

The following proposition gives the formula for the discount factor $D(t ; \varepsilon)$ such that welfare $V_{\tau}$ equals the present discounted value of future utility flows $u_{s}$, i.e.:

$$
V_{\tau}=\sum_{s=0}^{\infty} D(s ; \varepsilon) u_{\tau+s} \quad \text { with } \quad D(0 ; \varepsilon)=1
$$

Proposition 6 Assume $e^{-\lambda \varepsilon}\left(2-e^{-\theta \epsilon}\right)-e^{-\gamma \varepsilon} \neq 0$ and $\lambda>\theta$. (i) The additively separable function $V_{t}$ defined in equation (14) equals the solution to the recursion in equation (13) if and only if the discount factor equals

$$
D(t ; \varepsilon)=\frac{e^{-\gamma \varepsilon t}\left(e^{-\lambda \varepsilon}-e^{-\gamma \varepsilon}\right)+e^{-\lambda \varepsilon}\left(e^{-\lambda \varepsilon}\left(2-e^{-\theta \varepsilon}\right)\right)^{t} \theta \varepsilon}{e^{-\lambda \varepsilon}\left(2-e^{-\theta \varepsilon}\right)-e^{-\gamma \varepsilon}} .
$$

(ii) (a) $D(t ; \varepsilon)$ is positive, bounded and approaches 0 as $t \rightarrow \infty$.(b) $\sum_{s=0}^{\infty} D(s ; \varepsilon)$ is

\footnotetext{
${ }^{11}$ Replacing the first term on the right side of equation (13) by $\tilde{u}_{t} \equiv \sum_{t=0}^{\infty} \alpha^{t} u_{\tau+t}$ and defining $a(t)=\left(1-e^{-\theta \varepsilon}\right) e^{-\lambda \varepsilon t}$, results in equation (2) of Saez-Marti and Weibull (2005).
} 
bounded, so $V$ is bounded given that $u$ is bounded.

The term $e^{-\lambda \varepsilon}\left(2+e^{-\theta \varepsilon}\right)=\delta(1+b)$ appears in a formula used to prove Proposition 6. The following lemma establishes a bound on this term.

Lemma 2 The necessary and sufficient condition for $\delta b+\delta<1$ for all $\varepsilon \geq 0$ is $\lambda>\theta$.

Proof. Necessity: Using the definitions of $\delta$ and $b, \delta b+\delta=e^{-\lambda \varepsilon}\left(2-e^{-\theta \varepsilon}\right)$. The first order approximation of this expression, evaluated at $\varepsilon=0$, is $1+(\theta-\lambda) \varepsilon+o(\varepsilon)$. This expression is less than 1 if and only if $\lambda>\theta$. Sufficiency: Use $\frac{d\left(e^{-\lambda \varepsilon}\left(2-e^{-\theta \varepsilon}\right)\right)}{d \varepsilon}=$ $e^{-\lambda \varepsilon}\left((\theta+\lambda) e^{-\theta \varepsilon}-2 \lambda\right)$. If $\lambda>\theta$ then $(\theta+\lambda) e^{-\theta \varepsilon}-2 \lambda<2 \lambda\left(e^{-\theta \varepsilon}-1\right)<0$ so $e^{-\lambda \varepsilon}\left(2-e^{-\theta \varepsilon}\right)$ is decreasing in $\varepsilon$. Therefore, it is negative for all $\varepsilon \geq 0$ if and only if it is negative for $\varepsilon=0$.

Proof. Proposition 6. Substituting equation (14) into (13) gives

$$
V_{\tau}=\sum_{s=0}^{\infty} \alpha^{s} u_{\tau+s}+b \sum_{s=1}^{\infty} \delta^{s}\left(\sum_{k=0}^{\infty} D_{k} u_{\tau+s+k}\right)
$$

Making a change of variables, $t=s+k$ and then reversing the order of summation and simplifying yields

$$
V_{\tau}=u_{\tau}+\sum_{t=1}^{\infty}\left(\alpha^{t}+b \sum_{s=1}^{t} \delta^{s} D_{t-s}\right) u_{\tau+t} .
$$

Equating coefficients in equations (14) and (16) implies

$$
D_{t}=\alpha^{t}+b \sum_{s=1}^{t} \delta^{s} D_{t-s}
$$

with initial condition $D_{0}=1$. The manipulations above are valid because $\sum_{s=0}^{\infty} D(s ; \varepsilon)$ is bounded, as established in part (ii) below. 
An inductive proof establishes part (i). Setting $t=0$, the trial solution in equation (15) satisfies the initial condition $D_{0}=1$. Suppose that for $t \geq 0$, the trial solution solves the recursion (17) for $\tau \leq t$. I need to show that this hypothesis implies that the trial solution solves the recursion for $t+1$. The hypothesis implies

$$
D_{t+1}=\alpha^{t+1}+b \sum_{s=1}^{t+1} \delta^{s} D_{t+1-s}=\alpha^{t+1}+b \sum_{s=1}^{t+1} \delta^{s} \frac{\alpha^{t+1-s}(\delta-\alpha)+\delta b(\delta b+\delta)^{t+1-s}}{-\alpha+\delta b+\delta}
$$

Simplifying the last expression gives

$$
D_{t+1}=\frac{\alpha^{t+1}(\delta-\alpha)+\delta b(\delta b+\delta)^{t+1}}{-\alpha+\delta b+\delta}
$$

as was to be shown.

(iia) The facts that $\alpha<1$ and $(\delta b+\delta)<1$ (from Lemma 2) imply that $D_{t}$ is bounded and approaches 0 as $t \rightarrow \infty$. To show that $D_{t}>0$, consider three cases, where $\delta>\alpha$, where $\alpha>\delta>\frac{\alpha}{b+1}$, and where $\delta<\frac{\alpha}{b+1}$. In the first case, the numerator and denominator of $D_{t}$ are positive by inspection. In the second case, the denominator is positive. The numerator is positive iff

$$
L(t) \equiv\left(\frac{\delta b+\delta}{\alpha}\right)^{t}>\frac{\alpha-\delta}{\delta b}
$$

The function $L(t)$ is increasing in $t, L(0)=1$, and $\frac{\alpha-\delta}{\delta b}<1$ because $\delta>\frac{\alpha}{b+1}$; therefore, inequality (18) is satisfied. Consequently, the numerator of $D_{t}$ is positive, so $D_{t}$ is positive. In the third case, the denominator of $D_{t}$ is negative and the numerator is negative iff

$$
L(t) \equiv\left(\frac{\delta b+\delta}{\alpha}\right)^{t}<\frac{\alpha-\delta}{\delta b} .
$$

Here, $L(0)=1$ and is decreasing in $t$ and the right side of inequality (19) is greater than 1 , so the inequality is satisfied.

(iib) The fact that $D$ is the sum of two geometrically decreasing terms, means that it's infinite sum is bounded. The sum equals $\frac{1-\delta}{(1-\delta b-\delta)(1-\alpha)}$. 


\section{A.1.3 Deterministic lifetime, paternalistic altruism}

Here, in contrast to the case of exponentially distributed lifetimes, agents alive in a period are different: the older ones will die sooner than the younger ones. Agents therefore have different views about the future benefits of the public good. The social planner in a period is utilitarian: she maximizes the sum of the discounted utility of those currently alive, plus the value that those agents give to the utility of the not-yet born.

Agents live for $T \geq 1$ periods. ${ }^{12}$ For $T=2$, this model produces Laibson's (1997) $\beta, \delta$ model of quasi-hyperbolic discounting. Next period, the fraction $\left(1-\frac{1}{T}\right)$ of current agents will still be alive. The agents alive at $t=0$ discount their future utility using the selfish discount factor $e^{-r \varepsilon}$. The utilitarian social planner representing the agents alive at $t=0$ discounts their $t=1$ utility at $e^{-r \varepsilon}\left(1-\frac{1}{T}\right)$, which takes into account both the agents' impatience and the death of some of the agents alive at $t=0$. For $t \leq T-1$ the discount factor that this social planner uses to evaluate the future utility of agents currently alive is $e^{-r \varepsilon t}\left(1-\frac{t}{T}\right)$ and for $t \geq T$ the discount factor is 0 , because all of the original agents will have died.

With a constant population, $\frac{1}{T}$ new agents are born in each period. The agents alive in period 0 discount future generations' utility at $e^{-\lambda \varepsilon}$; for example, the weight that the agents alive in period 0 give to those born in year 1 is $\frac{e^{-\lambda \varepsilon}}{T}$, a factor that accounts for both the current agents' altruism and the fact that $\frac{1}{T}$ new agents arrive in each period. The agents who arrive in year 1 discount their own next period utility at $e^{-r \varepsilon}$, and because the agents alive in period 0 discount those agents' utility at $e^{-\lambda \varepsilon}$, the weight that the period 0 agents place on the $t=2$ utility of the agents who arrived at $t=1$ is $\frac{e^{-\lambda \varepsilon} e^{-r \varepsilon}}{T}$. Those alive at time 0 place the weight $\frac{e^{-\lambda \varepsilon i} e^{-r \varepsilon(t-i)}}{T}$ on the period $t>0$ utility of agents who arrive in period $i$, for $t-T<i \leq t$.

The weight that the $t=0$ social planner places on the utility flow at period $t \geq 0$, $D(t ; \varepsilon)$, equals the sum of the weights that the agents whom she represents place on the selfish and the altruistic components of their preferences. As noted above, the

\footnotetext{
${ }^{12}$ Sumaila and Walters (2005) propose a similar model, but their formulae account for birth but not for death. With constant population, the discount factor must account for both death and birth.
} 
selfish component of this discount factor has a different structure for $t<T$ and for $t \geq T$. Therefore, the function $D(t ; \varepsilon)$ also has a different structure for $t<T$ and for $t \geq T$.

In the discrete time setting, the weight that the social planner places on utility flow at a future time equals the sum of the weight attributed to agents currently alive and to those have not yet been born. This sum has a different structure, depending on whether $t \leq T-1$ or $t \geq T$. For $\lambda \neq r$ the discount factor is

$$
D(t ; \varepsilon)=\left\{\begin{array}{c}
e^{-r \varepsilon t}\left(1-\frac{t}{T}\right)+\frac{1}{T} \sum_{\tau=1}^{t} e^{-\lambda \varepsilon \tau} e^{-r \varepsilon(t-\tau)}= \\
\frac{1}{T}\left(\frac{e^{-\lambda \varepsilon}\left(e^{-\lambda \varepsilon t}-e^{-r \varepsilon t}\right)}{\left(e^{-\lambda \varepsilon}-e^{-r \varepsilon}\right)}+(T-t) e^{-r \varepsilon t}\right) \text { for } t \leq T-1 \\
\frac{1}{T} \sum_{\tau=t-T+1}^{t} e^{-\lambda \varepsilon \tau} e^{-r \varepsilon(t-\tau)}= \\
\frac{1}{T} e^{-\lambda \varepsilon(t+1-T)} \frac{e^{-\lambda \varepsilon T}-e^{-r \varepsilon T}}{e^{-\lambda \varepsilon}-e^{-r \varepsilon}} \text { for } t \geq T
\end{array}\right.
$$

For $T=2$, the discount factor at $t=1$ is $\frac{e^{-r \varepsilon}+e^{-\lambda \varepsilon}}{2}$ and the discount factor at $t>1$ is $\frac{1}{2} e^{-\lambda \varepsilon(t-1)}\left(e^{-\lambda \varepsilon}+e^{-r \varepsilon}\right)$. Defining $\beta=\frac{e^{-r \varepsilon}+e^{-\lambda \varepsilon}}{2} e^{\lambda \varepsilon}, \delta=e^{-\lambda \varepsilon}$ produces the $\beta, \delta$ model.

\section{A.2 The continuous time limit}

In the discrete time setting I do not need to distinguish between the number of units of time (e.g. years) and the number of periods. This distinction is important in using the discrete time model to obtain (by passing to a limit) the continuous time discount factors. That is, $t$ and $\tau$ refer to period indices in the discussion of discrete time models, and they refer to units of calendar time in the discussion of continuous time models; similarly, $T$ refers to the number of periods that the agent lives in the discrete time setting, and the number of units of time that she lives in the continuous time setting. For fixed $\varepsilon$, this notation does not present an issue, but here I want to consider the limiting case as $\varepsilon \rightarrow 0$. For this purpose, it is important to maintain the distinctions between the period index and units of time.

For this appendix (only) I use $\tau$ and $\Gamma$ to refer exclusively to units of time and 
$t$ and $T$ to refer exclusively to number of periods. If a period lasts $\varepsilon$ units of time, $\tau=t \varepsilon$ and $\Gamma=T \varepsilon$. Using these definitions, I set $t=\frac{\tau}{\varepsilon}$ and $T=\frac{\Gamma}{\varepsilon}$ and use the definitions in the last row of Table 3 to write the discrete time discount factors as a function of units of time (rather than number of periods) and the length of each period, $\varepsilon$. It is then a simple matter to take limits as $\varepsilon \rightarrow 0$.

\section{A.2.1 Exponentially distributed lifetime, paternalistic altruism}

Equation (12) can be simplified to

$$
D(t ; \varepsilon)=\frac{\left(\left(e^{-\lambda \varepsilon}-e^{-\gamma \varepsilon}\right) e^{-\gamma \varepsilon t}+\left(1-e^{-\theta \varepsilon}\right) e^{-\lambda \varepsilon}\left(e^{-\lambda \varepsilon t}-e^{-\gamma \varepsilon t}\right)\right)}{\left(e^{-\lambda \varepsilon}-e^{-\gamma \varepsilon}\right)}
$$

This simplification assumes that $\gamma \neq \lambda$; the case $\lambda=\gamma$ follows from L'Hospital's Rule.

Define $\mu(\epsilon)=\frac{b \delta}{\alpha-\delta}=\frac{\left(1-e^{-\theta \epsilon}\right) e^{-\lambda \epsilon}}{e^{-(r+\theta) \epsilon}-e^{-\lambda \epsilon}}$ and use L'Hospital's Rule to obtain $\lim _{\epsilon \rightarrow 0} \mu(\epsilon)=$ $\frac{\theta}{\lambda-\theta-r}=\frac{\theta}{\lambda-\gamma}$. Using this definition of $\mu$ and the last row of Table 3, equation (12), the discount function expressed as a function of time, rather than number of periods, is

$$
(1+\mu(\epsilon)) e^{-(r+\theta) \tau}-\mu(\epsilon) e^{-\lambda \tau}
$$

Letting $\epsilon \rightarrow 0$ gives the continuous time discount factor for calendar time $\tau$, equation (1). Ekeland and Lazrak (2010) obtain this formula directly (without the discrete time detour).

\section{A.2.2 Exponentially distributed lifetime, pure altruism}

Proof. Proposition 1 In order to establish the claim, denote the discount rate that the agent with pure altruism applies to future generations as $\lambda^{\prime}$ (instead of $\lambda$, the rate under paternalistic altruism). Define the function

$$
\xi(\varepsilon)=\frac{-\alpha+\delta(b+1)}{\varepsilon}=\frac{-e^{-\gamma \varepsilon}+e^{-\lambda^{\prime} \varepsilon}\left(2-e^{-\theta \varepsilon}\right)}{\varepsilon}
$$


and use $\lim _{\varepsilon \rightarrow 0} \xi(\varepsilon)=r+2 \theta-\lambda^{\prime}$. Also define $\phi(\varepsilon)=\frac{\ln \left(2-e^{\theta \varepsilon}\right)}{\varepsilon}$ and use $\lim _{\varepsilon \rightarrow 0} \phi(\varepsilon)=$ $\theta$. Finally, note that

$$
(b+1)^{t}=\left(2-e^{-\theta \varepsilon}\right)^{\frac{\tau}{\varepsilon}}=\exp \left(\tau \frac{\ln \left(2-e^{-\theta \varepsilon}\right)}{\varepsilon}\right)=e^{\tau \phi(\varepsilon)},
$$

so $\lim _{\varepsilon \rightarrow 0}\left(2-e^{-\theta \varepsilon}\right)^{\frac{t}{\varepsilon}}=e^{\tau \theta}$. With these definitions and the last row of Table 3 , the discrete time discount factor in equation (15) can be written

$$
\frac{e^{-\gamma \tau}\left(\frac{e^{-\lambda^{\prime} \varepsilon}-e^{-\gamma \varepsilon}}{\varepsilon}\right)+e^{-\lambda^{\prime} \varepsilon} \frac{\left(1-e^{-\theta \varepsilon}\right)}{\varepsilon} e^{-\lambda^{\prime} \tau} e^{\tau \phi(\varepsilon)}}{\xi} .
$$

Using the limiting expressions given above, the limit of this function as $\varepsilon \rightarrow 0$ gives the continuous time discount factor for the agent with pure altruism:

$$
D(t)=\frac{e^{-\gamma \tau}\left(\gamma-\lambda^{\prime}\right)+\theta e^{-\left(\lambda^{\prime}-\theta\right) \tau}}{\gamma+\theta-\lambda^{\prime}}
$$

The right side of equations (1) and (21) are equivalent if and only if $\lambda^{\prime}=\lambda+\theta$.

\section{A.2.3 Finite lifetime, paternalistic altruism}

Proof. Proposition 2 Define $\nu(\epsilon)=\frac{\delta-\rho}{\varepsilon}=\frac{\left(e^{-\lambda \epsilon}-e^{-r \epsilon}\right)}{\epsilon}$ and use $\lim _{\epsilon \rightarrow 0} \nu(\epsilon)=r-\lambda$. With this definition of $\nu$ and the last row of Table 3 , the discrete time discount factor for $t<\Gamma$ can be written

$$
\begin{gathered}
\frac{\varepsilon}{\Gamma(\delta-\rho)}\left(\delta\left(\delta^{t}-\rho^{t}\right)+(\Gamma-\tau) \rho^{t} \frac{(\delta-\rho)}{\varepsilon}\right) \\
=\frac{e^{-\lambda \varepsilon}\left(e^{-\lambda \tau}-e^{-r \tau}\right)}{\Gamma \nu}+\frac{(\Gamma-\tau)}{\Gamma} e^{-r \tau} .
\end{gathered}
$$

Taking the limit as $\varepsilon \rightarrow 0$ and rearranging the resulting expression produces the first line of equation (2). 
For $t \geq \Gamma$ the discount factor is

$$
\frac{\varepsilon}{\Gamma(\delta-\rho)} e^{\lambda(\Gamma-\tau-\varepsilon)}\left(e^{-\lambda \Gamma}-e^{-r \Gamma}\right)
$$

Taking the limit as $\varepsilon \rightarrow 0$ produces the second line of equation (2).

\section{B Proofs and technical details}

This appendix provides the equilibrium conditions for cases not considered in the text (B.1), discusses sufficiency (B.2), provides the proofs of Lemma 2 and Proposition 3 (B.3) and the proof of Propositions 4 and 5, and Corollaries 2, 3, and 4, and the justification for Remark 1 (B.4).

\section{B.1 Equilibrium conditions for other cases}

There are two cases under exponentially distributed lifetime and a single case with a deterministic lifetime, because in the former but not in the latter, $\lim _{t \rightarrow \infty} \eta(t)$ depends on whether $\lambda<r$ or $\lambda>r$. For the exponential case with $0<\lambda \leq r$, and using the differentiability of $J(S)$ (already assumed in deriving the problem comprised of (6) and ), a necessary condition for the MPE is that

$$
x_{t}=\chi\left(S_{t}\right) \equiv \arg \max \left(u\left(S_{t}, x_{t}\right)-K\left(S_{t}\right)+J_{S}(S) F(S, x)\right)
$$

and that $J(S)$ satisfy the dynamic programming equation ${ }^{13}$

$$
\lambda J(S)=\left(u(S, \chi(S))-K(S)+J_{S}(S) F\left(S, \chi\left(S_{t}\right)\right)\right) .
$$

For the exponential case with $\lambda>r$, where $\lim _{t \rightarrow \infty} \eta(t)=\gamma$, the fictitious control

\footnotetext{
${ }^{13}$ Appendix B.2 explains why these necessary conditions, together with the definition in equation (7), are also sufficient for a MPE.
} 
problem is

$$
J(S)=\max \int_{0}^{\infty} e^{-\gamma t}\left(u\left(S_{t}, x_{t}\right)-K\left(S_{t}\right)\right) d \tau \quad \text { subject to } \dot{S}=F(S, x),
$$

with the side condition (definition):

$$
K\left(S_{t}\right) \equiv \int_{0}^{\infty} D(\tau)(\eta(\tau)-\gamma) u\left(S_{\tau}^{*}, \chi\left(S_{\tau}^{*}\right)\right) d \tau
$$

Equation (1) and the first line of equation (3) imply $D(t)(\eta(t)-\gamma)=-\theta e^{-\lambda t}$ so equation (25) simplifies to

$$
K\left(S_{t}\right)=-\theta \int_{0}^{\infty} e^{-\lambda \tau} u\left(S_{t+\tau}^{*}, \chi\left(S_{t+\tau}^{*}\right)\right) d \tau
$$

The integral in equation (26) is the present discounted value of the equilibrium future flow of payoff, computed using the discount rate $\lambda$. Thus, $-K\left(S_{t}\right)$ is an annuity, which if received in perpetuity and discounted at $\theta$ (the constant birth = death rate), equals the value of this future stream of payoff. The flow payoff in the fictitious control problem equals the flow playoff in the original model, plus this annuity. A necessary condition for the MPE is that

$$
x_{t}=\chi\left(S_{t}\right) \equiv \arg \max \left(u\left(S_{t}, x_{t}\right)-K\left(S_{t}\right)+J_{S}(S) F(S, x)\right),
$$

and that $J(S)$ satisfy the dynamic programming equation

$$
\gamma J(S)=\left(u(S, \chi(S))-K(S)+J_{S}(S) F\left(S, \chi\left(S_{t}\right)\right)\right)
$$

For agents with deterministic lifetime $T$, the function $K$ has a slightly different form than above. Using equation (4) of Karp (2007) and equations (4) and (2), $-K$ 
equals $^{14}$

$$
-K\left(S_{t}\right) \equiv(\lambda-r) \int_{0}^{T} \frac{(T-\tau)}{T} e^{-r \tau} U\left(S_{t+\tau}^{*}, \chi\left(S_{t+\tau}^{*}\right)\right) d \tau .
$$

The integral on the right side of definition (29) is the present value, discounted at the selfish rate $r$, of the payoff that those alive at $t$ receive from the flow $U\left(S_{t+\tau}^{*}, \chi\left(S_{t+\tau}^{*}\right)\right)$ over $[t, T+t]$. Over that interval, the number of agents remaining from the time $t$ population decreases linearly. Again, we can interpret $-K$ as an annuity, which if received in perpetuity and discounted at the rate $(\lambda-r)$, equals the present value to those currently alive of the program $U\left(S_{t+\tau}^{*}, \chi\left(S_{t+\tau}^{*}\right)\right)$.

The dynamic programming equation in this case is

$$
\lambda J(S)=\max _{x}\left(U\left(S_{t}, x_{t}\right)-K\left(S_{t}\right)+J_{S}(S) f(S, x)\right)
$$

with the annuity $K$ given by equation (29).

\section{B.2 Sufficiency}

The discussion of sufficiency in Karp (2007) is misleading, and I take this opportunity to clarify it. The endogeneity of the function $K(S)$, and the resulting difficulty in determining its curvature, makes it difficult to apply standard sufficiency conditions for optimal control problems, to the fictitious control problem defined by equations (24) and (25). However, the fictitious control problem is merely a device for describing the equilibrium to the sequential game induced by non-constant discounting; for that purpose, we use only the necessary conditions to the fictitious problem. The maximization problem in equation (21) of Karp (2007) is a statement of the problem for the planner in a particular period in a discrete time setting, under the assumption of Markov perfection. Equation (5) of that paper (equivalently, equation (28) above) is the limiting form of the discrete time condition, as the length of a period of commitment goes to 0 . Therefore, provided that we are willing to restrict attention

\footnotetext{
${ }^{14} \operatorname{Karp}(2007)$ sets up the problem using a discount rate $r(t)$ for $t \leq T$ and $r(t)=\bar{r}$ for $t \geq T$. The results for the case of exponentially distributed lifetime use the limiting case as $T \rightarrow \infty$. In the OLG model with finitely lived agents, $T$ is finite and $\bar{r}=\lambda$.
} 
to the limiting game (as the length of a period goes to zero in the discrete time game), and provided that the value function is differentiable, a sufficient condition for the MPE is that the control rule satisfy equation (27) above, and that the value function satisfy the DPE (28).

The primitive functions of some interesting optimal control problems do not have the curvature need to satisfy familiar sufficient conditions. Sufficiency in optimal control problems is therefore sometimes a difficult issue, and the analysis sometimes proceeds without reference to sufficiency. The difficulty arises because sufficiency is a global property in optimal control problems. In contrast, sufficiency is a much simpler issue in the type of sequential game induced by non-constant discounting and the requirement of Markov perfection. In this game, each of the succession of social planers chooses a single action; given her beliefs about successors' policy function, each policy maker thus solves a static optimization problem. Because each of the policymakers treats the functions $J(S)$ and $K(S)$ as predetermined (although they are endogenous to the game), sufficiency requires (in the limit as $\varepsilon \rightarrow 0$ ) only that $x=\chi(S)$ maximizes $\left(U\left(S_{t}, x_{t}\right)+J_{S}(S) f(S, x)\right)$.

For the climate model, $U$ is concave in the control and $f$ is linear, so the necessary condition to $\max _{x}\left(U\left(S_{t}, x_{t}\right)+J_{S}(S) f(S, x)\right)$ is also sufficient.

\section{B.3 Proof of Lemma 1 and Proposition 3}

Proof. (Lemma 1) For small $\varepsilon>0$ define the $\tau$ as the smallest time beyond which $\left|\frac{U(t)-U_{\infty}}{U_{\infty}}\right| \leq \varepsilon$. That is

$$
\tau=\inf _{t}\left\{t:\left|\frac{U(\tau)-U_{\infty}}{U_{\infty}}\right| \leq \varepsilon \forall \tau \geq t\right\}
$$

Note that $\tau<\infty$. Use

$$
\begin{gathered}
\lim _{\lambda \rightarrow 0}\left(\frac{\int_{0}^{\infty} D(t) U(t) d t}{\frac{U_{\infty}}{\lambda}}\right) \\
=\lim _{\lambda \rightarrow 0} \frac{\lambda\left[\left(\int_{0}^{\tau} D(t) U(t) d t\right)+\left(U_{\infty} \int_{\tau}^{\infty} D(t) d t\right)+\left(\int_{\tau}^{\infty} U(t) D(t) d t-U_{\infty} \int_{\tau}^{\infty} D(t) d t\right)\right]}{U_{\infty}} .
\end{gathered}
$$


Consider each of the three terms on the right side of this equation. The fact that $\tau<\infty$ implies that

$$
\lim _{\lambda \rightarrow 0} \lambda \int_{0}^{\tau} D(t) U(t) d t=0
$$

A calculation confirms that

$$
\int_{\tau}^{\infty}\left(\left(\frac{\lambda-r}{\lambda-\gamma}\right) e^{-\gamma t}-\frac{\theta}{\lambda-\gamma} e^{-\lambda t}\right) d t=\frac{-e^{-\gamma \tau} \lambda^{2}+e^{-\gamma \tau} \lambda r+\theta e^{-\lambda \tau} \gamma}{(-\lambda+\gamma) \gamma \lambda}
$$

Taking the limit as $\lambda \rightarrow 0$ of this expression, implies that the second term on the second line of equation (31) equals $\frac{\theta}{\gamma}$. By definition of $\tau$,

$$
\left|\frac{\int_{\tau}^{\infty} U(t) D(t) d t-U_{\infty} \int_{\tau}^{\infty} D(t) d t}{U_{\infty}}\right|<\varepsilon \int_{\tau}^{\infty} D(t) d t
$$

The limit as $\lambda \rightarrow 0$ of the last expression is $\varepsilon \frac{\theta}{\gamma}$.

Proof. (Proposition 3) I first derive the necessary and sufficient condition, for general $n$, that must be satisfied at a stable steady state in a differentiable MPE. I then specialize to $n=1$ and show that the boundary of the open interval of states that satisfies this condition is arbitrarily close to the UMSS for $\lambda$ close to 0 . Because I am interested in the case where $\lambda$ is small, I assume throughout that $\lambda<r$.

Denote agent $i$ 's policy function as $\chi(S)$ and the aggregate decision as $\Psi \equiv n \chi$, so $\Psi^{\prime}=n \chi^{\prime}$. Define

$$
z=\left(f_{S}+f_{\Psi} \Psi^{\prime}\right)_{\mid \infty}
$$

where the subscript $\infty$ denotes that the function is evaluated at a steady state. Stability requires $z<0$. For $S_{t} \approx S_{\infty}$, a first order approximation gives

$$
S_{t+\tau}=e^{z \tau} S_{t}+S_{\infty}\left(1-e^{z \tau}\right)+o\left(S_{t}-S_{\infty}\right) \Longrightarrow \frac{d S_{t+\tau}}{d S_{t}} \approx e^{z \tau}
$$


for $\tau \geq 0$. Equation (7) implies

$$
\begin{gathered}
K^{\prime}\left(S_{t}\right)= \\
(r-\lambda) \int_{0}^{\infty} e^{-\gamma \tau}\left(u_{S}\left(S_{t+\tau}, \chi\left(S_{t+\tau}\right)\right)+u_{x}\left(S_{t+\tau}, \chi\left(S_{t+\tau}\right)\right) \chi^{\prime}\left(S_{t+\tau}\right)\right) \frac{d S_{t+\tau}}{d S_{t}} d \tau .
\end{gathered}
$$

Using equation (32) and evaluating equation (33) at $S_{t}=S_{\infty}$ gives

$$
\begin{gathered}
K^{\prime}\left(S_{\infty}\right)=(r-\lambda)\left(u_{S}+u_{x} \chi^{\prime}\right)_{\mid \infty} \int_{0}^{\infty} e^{-\gamma t} e^{z t} d t \\
=\frac{(r-\lambda)\left(u_{S}+u_{x} \chi^{\prime}\right)_{\left.\right|_{\infty}}}{\gamma-z}=\frac{(r-\lambda)\left(u_{S}+u_{x} \frac{\Psi^{\prime}}{n}\right)_{\left.\right|_{\infty}}}{\gamma-z} .
\end{gathered}
$$

The Hamiltonian corresponding to the fictitious optimal control problem in equation (6) is

$$
H=u(S, x)-K(S)+\mu f\left(S, x+\frac{n-1}{n} \Psi(S)\right),
$$

where $\mu$ is the current value costate variable. The necessary conditions for optimality are

$$
u_{x}+\mu f_{x}=0 \Longrightarrow \mu=-\frac{u_{x}}{f_{x}} \text { and } \dot{\mu}=\lambda \mu-\left(u_{S}-K^{\prime}+\mu\left(f_{S}+\frac{n-1}{n} f_{x} \Psi^{\prime}\right)\right) .
$$

Using the first necessary condition and evaluating the costate equation at a steady state (setting $\dot{\mu}=0$ ) gives the condition

$$
\begin{gathered}
{\left[-u_{S}+K^{\prime}+\frac{u_{x}}{f_{x}}\left(f_{S}+\frac{n-1}{n} f_{x} \Psi^{\prime}-\lambda\right)\right]_{\mid \infty}=} \\
{\left[-u_{S}+\frac{(r-\lambda)\left(u_{S}+u_{x} \frac{\Psi^{\prime}}{n}\right)}{\gamma-z}+\frac{u_{x}}{f_{x}}\left(f_{S}+\frac{n-1}{n} f_{x} \Psi^{\prime}-\lambda\right)\right]_{\mid \infty}=0,}
\end{gathered}
$$

where the first equality uses equation (34). Using the definition of $z$ and rearranging the second line of equation (35) implies that $\Psi^{\prime}=\Psi^{\prime}\left(S_{\infty}\right)$ is a solution to the quadratic equation

$$
Q \times\left(\Psi^{\prime}\right)^{2}+L \times \Psi^{\prime}+C=0
$$


with

$$
\begin{gathered}
Q \equiv u_{x} \frac{n-1}{n} f_{x} \\
L \equiv\left((r-\lambda) \frac{1}{n}+\frac{n-1}{n}\left(\gamma-f_{S}\right)-\left(\left(f_{S}-\lambda\right)-\frac{u_{S}}{u_{x}} f_{x}\right)\right) u_{x} \\
C \equiv\left(-\lambda-\theta+f_{S}\right) u_{S}+\frac{u_{x}}{f_{x}}\left(f_{S}-\lambda\right)\left(\gamma-f_{S}\right)
\end{gathered}
$$

Hereafter I set $n=1$, so

$$
\begin{gathered}
\Psi_{L_{\infty}}^{\prime}=\frac{\left(\lambda+\theta-f_{S}\right) \frac{u_{S}}{u_{x}}-\frac{1}{f_{x}}\left(f_{S}-\lambda\right)\left(\gamma-f_{S}\right)}{r-f_{S}+\frac{u_{S}}{u_{x}} f_{x}} \Longrightarrow \\
z=f_{S}+f_{x} \frac{\left(\lambda+\theta-f_{S}\right) \frac{u_{S}}{u_{x}}-\frac{1}{f_{x}}\left(f_{S}-\lambda\right)\left(\gamma-f_{S}\right)}{r-f_{S}+\frac{u_{S}}{u_{x}} f_{x}}= \\
\frac{\theta\left(\frac{u_{S}}{u_{x}}-\frac{f_{S}}{f_{x}}\right) f_{x}+\lambda\left(\frac{u_{S}}{u_{x}}-\frac{f_{S}}{f_{x}}+\frac{\gamma}{f_{x}}\right) f_{x}}{r+\left(\frac{u_{S}}{u_{x}}-\frac{f_{S}}{f_{x}}\right) f_{x}} .
\end{gathered}
$$

The UMSS is a solution to

$$
\left(\frac{u_{S}}{u_{x}}-\frac{f_{S}}{f_{x}}\right) u_{x}=0 .
$$

I define the state and the control variables so that $u_{S}<0$ and $u_{x}<0$. For example, in the climate model, $S$ is the stock of atmospheric carbon and $x$ is the level of abatement, so the flow of utility is decreasing in both variables. These definitions (the state variable is a "bad" and the action is costly) mean that the model is sensible if and only if $f_{x}<0$ (so that incurring a cost reduces the public bad). Given the concavity of the static optimization problem (which determines the UMSS), a stock level slightly greater than the UMSS satisfies

$$
\left(\frac{u_{S}}{u_{x}}-\frac{f_{S}}{f_{x}}\right) u_{x}=\varepsilon<0 \text { or }\left(\frac{u_{S}}{u_{x}}-\frac{f_{S}}{f_{x}}\right)=\frac{\varepsilon}{u_{x}}>0,
$$

for $\varepsilon$ small in absolute value. Such a stock level yields approximately the maximum steady state level of utility. (Given that the costly action $x$ reduces the stock, it would never be part of an equilibrium to drive the stock below the optimal static level.) 
Using equation (38) in (37) gives

$$
z=\frac{\theta \frac{\varepsilon}{u_{x}} f_{x}+\lambda\left(\frac{\varepsilon}{u_{x}}+\frac{\gamma}{f_{x}}\right) f_{x}}{r+\frac{\varepsilon}{u_{x}} f_{x}} .
$$

The denominator is positive for small $\varepsilon$. For $\varepsilon$ small in absolute value (so that $\left.\frac{\varepsilon}{u_{x}} f_{x}+\gamma>0\right)$, the numerator is negative if and only if

$$
\frac{-\theta \frac{\varepsilon}{u_{x}} f_{x}}{\left(\frac{\varepsilon}{u_{x}} f_{x}+\gamma\right)}>\lambda
$$

i.e. if and only if $\lambda$ is sufficiently small, as was to be shown.

\section{B.4 The constant MPE in the linear-in-state model}

The first part of this appendix provides proofs Propositions 4 and 5 and Corollaries 2, 3, and 4. The second part justifies Remark 1 .

\section{B.4.1 Proofs: linear-in-state}

Because $P$ is of full rank, $B=P \Lambda P^{-1}$. In a symmetric MPE, i.e. where all tribes emit $x(t)=\chi(t), \dot{S}(t)=c+B S(t)+C n \chi(t)$. Here, the equilibrium value of the state $t$ periods in the future, given the current value $S_{0}$ is: $S_{t}=P e^{\Lambda t} P^{-1} S_{0}+H(t)$. I note for future use that if $\chi$ is a constant, then $H(t)=P \Omega(t) P n^{-1}(c+C n \chi)$. If all eigenvalues are negative, $\Omega(t)$ is a diagonal matrix with element $\frac{\exp \left(\Lambda_{i} t-1\right)}{\Lambda_{i}}$ in the $i$ 'th diagonal position. However, for the calibration in Section B.4.2 I want to consider the case where the largest eigenvalue, denoted $\Lambda_{1}$, equals 0 , and others are negative. In this case, $\Omega(t)$ is a diagonal matrix with first diagonal element equal to $t$ and the other $i$ diagonal elements equal to $\frac{\exp \left(\Lambda_{i} t-1\right)}{\Lambda_{i}}$.

Proof. (Proposition 4) Given that the policy maker in tribe $i$ at time $t$ expects future emissions to be independent of the state, using the flow payoff $v(x ; n)-\frac{\kappa s}{n}=$ 
$\frac{1}{n}\left[v(n x ; 1)-\kappa i_{1}^{\prime} S\right]$ and equation $(7)$, the annuity function is :

$$
\begin{gathered}
K\left(S_{t}, t\right)=\frac{(r-\lambda)}{n} \int_{0}^{\infty} e^{-\gamma \tau}\left[\left(v(n \chi(t+\tau) ; 1)-\kappa i_{1}^{\prime} S_{t+\tau}\right)\right] d \tau \\
=\frac{(r-\lambda)}{n} \int_{0}^{\infty} e^{-\gamma \tau}\left[\left(v(n \chi(t+\tau) ; 1)-\kappa i_{1}^{\prime}\left(P e^{\Lambda \tau} P^{-1} S_{t}+H(t+\tau)\right)\right)\right] d \tau .
\end{gathered}
$$

From this formula, it is apparent that $K$ is linear in $S, K\left(S_{t}, t\right)=q_{0, t}+q^{\prime} S_{t}$, with the gradient $q^{\prime}$ given by the first equation in (8). If future policies are constant, then $q_{0, t}$ is a constant, $q_{0}$.

Using $K=q_{0, t}+q^{\prime} S$ and the utility flow $v(x ; n)-\frac{\kappa s}{n}$ in equation (6), produces the dynamic programming equation (DPE)

$$
\begin{gathered}
\lambda J(S, t)=\max _{x}\left\{v(x ; n)-\frac{\kappa}{n} i_{1}^{\prime} S\right. \\
\left.-\left(q_{0, t}+q^{\prime} S\right)+J_{S}^{\prime}(S, t)\left[c+B S+C\left(x+(n-1) \chi_{t}\right)\right]\right\} .
\end{gathered}
$$

Because this problem is linear in the state, the obvious trial solution is a linear function, $J(S, t)=g_{0, t}+g_{t}^{\prime} S$. Using this trial solution, the DPE becomes

$$
\begin{gathered}
\lambda\left(g_{0, t}+g^{\prime} S\right)=\max _{x}\left\{v(x ; n)+g_{t}^{\prime} C x\right\} \\
-\frac{\kappa}{n} i_{1}^{\prime} S-\left(q_{0, t}+q^{\prime} S\right)+g_{t}^{\prime}[c+B S+C(n-1) \chi] .
\end{gathered}
$$

The first order condition (which is sufficient due to concavity of $v$ ) is

$$
\frac{\partial v(x ; n)}{\partial x}+g_{t}^{\prime} C=0
$$

The solution, $x^{*}$, possibly depends on time, but is independent of the state. Substituting the optimal flow payoff into the the DPE gives the maximized DPE

$$
\begin{gathered}
\lambda\left(g_{0, t}+g_{t}^{\prime} S\right)=v\left(x^{*} ; n\right)+g^{\prime} C x^{*} \\
-\frac{\kappa}{n} i_{1}^{\prime} S-\left(q_{0, t}+q^{\prime} S\right)+g_{t}^{\prime}[c+B S+C(n-1) \chi] .
\end{gathered}
$$


Equating coefficients of $S$ gives

$$
\lambda g_{t}^{\prime}=-\frac{\kappa}{n} i_{1}^{\prime}-q^{\prime}+g_{t}^{\prime} B
$$

Because $B$ and $q^{\prime}$ are constants, $g_{t}^{\prime}$ is also a constant, $g^{\prime}$, given by the last equation in (8). Thus, $q_{0}$, and $g_{0}$ are also constants.

In summary, regardless of planner $i, t$ 's beliefs about other planners' state-independent policies, planner $i, t$ 's optimal policy is the constant given by $x=\arg \max _{x}\left\{v(x ; n)+g^{\prime} C x\right\}$. Because $g$ is independent of other planners' policies, the equilibrium policy is dominant both respect to actions by future planners in one's own tribe, and by all current and future planners in other tribes.

Proof. (Corollary 2) For the log-linear model, where $v(x ; n)=\frac{\alpha}{n} \ln (n x)$, the first order condition for the problem in (9) is $\frac{\alpha}{n x}+g^{\prime} C=0 \Longrightarrow x=-\frac{\alpha}{n g^{\prime} C} \Longrightarrow$ $n x=X=-\frac{\alpha}{g^{\prime} C}=\frac{-\alpha n}{\kappa\left(i_{1}^{\prime}-(r-\lambda) \tilde{q}^{\prime}\right)(B-\lambda I)^{-1} C}$. Emissions are positive $\left(g^{\prime} C<0\right)$, so aggregate emissions are an increasing linear function of $n$. For the quadratic model, where $v(x ; n)=a x-\frac{d n}{2} x^{2}$, the first order condition for the problem in (9) is $a-d n x+g^{\prime} C=0 \Longrightarrow x=\frac{a+g^{\prime} C}{d n} \Longrightarrow n x=X=\frac{a+g^{\prime} C}{d}$. Using $\frac{d\left(g^{\prime} C\right)}{d n}=$ $-\frac{\kappa}{n^{2}}\left(i_{1}^{\prime}-(r-\lambda) \tilde{q}^{\prime}\right)(B-\lambda I)^{-1} C>0$, aggregate emissions is an increasing strictly concave function of $n$.

Proof. (Proposition 5) The first order condition for the problem in equation (9) is $\frac{d v(x ; n)}{d n}=-g(n)^{\prime} C$, where I make the dependence of $g$ on $n$ explicit for emphasis. By concavity of $v$, this first order condition is sufficient. Using the definition $v(x ; n)=$ $\frac{1}{n} v(n x ; 1)$, and the chain rule, I have $\frac{d v(x ; n)}{d n}=\frac{1}{n} \frac{d v(n x ; 1)}{d(n x)} \frac{d n x}{d x}=\frac{d v(X(n) ; 1)}{d X}$. Using this relation in the first order condition gives $\frac{d v(X(n) ; 1)}{d X}=-g(n)^{\prime} C$. Using the definition of $g(n)^{\prime}$ from equation (8) in this first order condition gives equation (10).

By inspection, the absolute value of the elasticity of this tax with respect to $n$ is 1. In order to obtain equation (11), use

$$
\begin{gathered}
\frac{d \tau}{d \lambda}=-\frac{\kappa}{n} \frac{d\left[\left(i_{1}^{\prime}-(r-\lambda) \tilde{q}^{\prime}\right)(B-\lambda I)^{-1} C\right]}{d \lambda}= \\
-\frac{\kappa}{n}\left[\tilde{q}^{\prime}(B-\lambda I)^{-1} C+\left(i_{1}^{\prime}-(r-\lambda) \tilde{q}^{\prime}(B-\lambda I)^{-2} C\right)\right]= \\
-\frac{\kappa}{n}\left[\tilde{q}^{\prime}+\left(i_{1}^{\prime}-(r-\lambda) \tilde{q}^{\prime}(B-\lambda I)^{-1}\right)\right](B-\lambda I)^{-1} C .
\end{gathered}
$$


Multiplying this expression by $-\frac{\lambda}{\tau}$ to convert to an absolute value elasticity, gives equation (11).

Proof. (Corollary 3) For the scalar case, $\tilde{q}=\frac{1}{\gamma-\hat{B}}$. Using this result in equation (10) produces the formula for $\tau^{*}$ in the scalar case. Straightforward calculation establishes the other claims in the corollary.

Proof. (Corollary 4) Part (i) is immediate from Corollary 3. To establish Part (ii), recall that the Woodbury Matrix Identify states that $(A+U D V)^{-1}=A^{-1}-$ $A^{-1} U\left(D^{-1}+V A^{-1} U\right)^{-1} V A^{-1}$. Let $A=-\lambda I, U=P, V=P^{-1}$ and $D=\Lambda$. First consider $\lim _{\Lambda^{\min } \rightarrow 0} \tau^{*}$. Suppose that $\Lambda^{-1}$ exists, i.e. $\Lambda^{\max }<0$. Applying the Woodbury Matrix Identity gives

$$
\begin{gathered}
\left(-\lambda I+P \Lambda P^{-1}\right)^{-1}=\frac{1}{-\lambda} I-\frac{1}{-\lambda} P\left(\Lambda^{-1}-\frac{1}{\lambda} P^{-1} P\right) P^{-1} \frac{1}{-\lambda} \\
=\frac{1}{-\lambda} I+\frac{1}{\lambda^{2}} P\left(\Lambda^{-1}-\frac{1}{\lambda} I\right) P^{-1} .
\end{gathered}
$$

Using $P\left(\Lambda^{-1}-\frac{1}{\lambda} I\right) P^{-1} \rightarrow 0 \times I$ as $\Lambda^{\text {min }} \rightarrow 0$ (from below), I have

$$
\lim _{\Lambda^{\min } \rightarrow 0}\left(-\lambda I+P \Lambda P^{-1}\right)^{-1}=\frac{1}{-\lambda} I .
$$

I also have

$$
\begin{gathered}
\lim _{\Lambda^{\min } \rightarrow 0} \tilde{q}=\lim _{\Lambda^{\min } \rightarrow 0} \int_{0}^{\infty} i_{1}^{\prime} P e^{-(\gamma I-\Lambda) \tau} P^{-1} d \tau \\
=\lim _{\Lambda^{\min } \rightarrow 0} i_{1}^{\prime} P \int_{0}^{\infty} e^{-(\gamma I-\Lambda) \tau} d \tau P^{-1}=i_{1}^{\prime} P \frac{1}{\gamma} P^{-1}=i_{1}^{\prime} I \frac{1}{\gamma} .
\end{gathered}
$$

Putting these results together

$$
\begin{gathered}
\lim _{\Lambda^{\min } \rightarrow 0} \tau^{*}=-\frac{\kappa}{n} \lim _{\Lambda^{\min } \rightarrow 0}\left(i_{1}^{\prime}-(r-\lambda) \tilde{q}^{\prime}\right)(B-\lambda I)^{-1} C \\
=-\frac{\kappa}{n} i_{1}^{\prime}\left(1-\frac{r-\lambda}{\gamma}\right) \frac{1}{-\lambda} I=\frac{\kappa}{n \lambda}\left(\frac{r+\theta-r+\lambda}{r+\theta}\right)=\frac{\kappa}{n \lambda}\left(\frac{\theta+\lambda}{r+\theta}\right) .
\end{gathered}
$$

Now consider $\lim _{\Lambda^{\max } \rightarrow-\infty} \tau^{*}$. Using the definition of $\tilde{q}, \lim _{\Lambda^{\max \rightarrow-\infty}} \tilde{q}=0$. In addition, $(B-\lambda I)^{-1}=\left(P \Lambda P^{-1}-\lambda I\right)^{-1} \rightarrow 0 \times I$ as $\Lambda^{\max } \rightarrow-\infty$. Thus, $\lim _{\Lambda^{\max } \rightarrow-\infty} \tau^{*}=0$. 


\section{B.4.2 The Gerlagh and Liski (2012) calibration}

Gerlagh and Liski (2012) calibrate a four dimensional discrete time model that produces relations among emissions, stock accumulation, and economic damages that are similar to those in DICE (Nordhaus 2008). Here I explain how I modify their model to fit the continuous time setting. The state variable is $S=\left(D, L_{1}, L_{2}, L_{3}\right)$. These variables correspond to: (damages $(D)$, carbon stocks in the atmosphere + upper ocean $\left(L_{1}\right)$, carbon stock in the biosphere $\left(L_{2}\right)$ and carbon stock in the deep ocean $\left(L_{3}\right)$. Using a unit of time equal to a decade, their calibration "almost" produces $D_{1}=(1-\varepsilon) D_{0}+\varepsilon \pi L_{1,0}$, with the adjustment parameter $\varepsilon=0.156$ and the damage parameter $\pi=0.0122 .{ }^{15}$ Let the parameters corresponding to an annual step be $\tilde{\varepsilon}$ and $\tilde{\pi}$. After 10 years (holding $L_{1, t}$ constant at $L_{1,0}$ ) $D_{10}=$ $(1-\tilde{\varepsilon})^{10} D_{0}+\sum_{j=0}^{9}(1-\tilde{\varepsilon})^{j} \tilde{\varepsilon} \tilde{\pi} L_{1,0} . \quad$ I convert from a decadal to an annual time step, using $(1-\tilde{\varepsilon})^{10}=1-\varepsilon \Longrightarrow \tilde{\varepsilon}=0.017$; for the annual time step I replace $\varepsilon \pi$ with $\tilde{\varepsilon} 0.0122=2.05 \times 10^{-4}$.

This procedure gives the parameters for the first difference equation, with an annual time step. I obtain the remaining parameters in the dynamic system (with the assistance of personal communication) from the supplementary material that accompanies their paper. The equation of motion is $S_{t}=\tilde{B} S_{t-1}+\tilde{C} x_{t}$, with

$$
\tilde{B}=\left[\begin{array}{cccc}
0.9831827374 & 2.051706037 \times 10^{-4} & 0 & 0 \\
0 & 0.963084 & 0.027280 & 0.003262 \\
0 & 0.025115 & 0.972720 & 0.000000 \\
0 & 0.011801 & 0.000000 & 0.996738
\end{array}\right] ; \quad \tilde{C}=\left[\begin{array}{l}
0 \\
1 \\
0 \\
0
\end{array}\right]
$$

At a constant aggregate emissions rate of $x$ (as in the constant symmetric equilibrium), and given an initial condition $S_{0}$, the state in the next period is $S_{1}=$

\footnotetext{
${ }^{15}$ In their model, current damages depend on lagged damages and the current atmospheric stock, so they have $D_{t}=(1-\varepsilon) D_{t-1}+\varepsilon \pi L_{1, t}$. I have current damage depend on lagged damage and lagged atmospheric stock. With their formulation, the utility flow depends on two state variables, $D$ and $L_{1}$. I would need to introduce an additional state variable, a weighted sum of $D$ and $L_{1}$ in order for the utility flow to depend on a single state. My alternative is to have current damages depend on lagged $L_{1}$.
} 
$\tilde{B} S_{0}+\tilde{C} x$. Given these numerical values, I find the eigenvalues and eigenvectors to write $\tilde{B}=\tilde{P} \tilde{\Lambda} \tilde{P}^{-1}$.

In the continuous time system, the stock after one year of constant emissions is $S_{1}=P e^{\Lambda} P^{-1} S_{0}+P \Omega P^{-1} C x$. Given the same initial condition and the same level of emissions, the state at the end of the period should be the same in the discrete and the continuous time systems. This condition requires

$$
P e^{\Lambda} P^{-1}=\tilde{P} \tilde{\Lambda} \tilde{P}^{-1} \text { and } P \Omega^{-1} P^{-1} C=\tilde{C} .
$$

The first equation is satisfied by setting $P=\tilde{P}$ and $e^{\Lambda}=\tilde{\Lambda}$. With these values, I solve the second equation to obtain $C=P \Omega^{-1} P^{-1} \tilde{C} .{ }^{16}$ With $P, \Lambda, C$ and values for $r, \gamma, \lambda$, I compute the formula for the elasticity in equation (11).

With their calibration and $r=0.2=\theta$, Table 4 shows that for $\lambda<0.0133$, the elasticity of the tax with respect to $\lambda$ is less than the elasticity with respect to $n$ (always equal to 1 ). The relation reverses for larger $\lambda$; for $\lambda$ close to its upper bound (in this setting, $r$ ), the elasticity of the tax with respect to altruism is nearly five times as large as the elasticity with respect to $n$. This example shows the flexibility of the tools developed here, and the importance of non-monotonic dynamics.

\begin{tabular}{|l|l|l|l|l|l|l|}
\hline$\lambda$ & .006 & .01 & 0.0133 & .016 & .018 & 0.02 \\
\hline$\varepsilon$ & 0.29 & 0.6 & 1 & 1.94 & 2.89 & 4.76 \\
\hline
\end{tabular}

Table 4: Relation between $\lambda$ and $\varepsilon$ in model adapted from Gerlagh and Liski (2012)

\footnotetext{
${ }^{16}$ In the discrete time model, all emissions go first to $L_{1}$ the upper atmosphere and shallow ocean $(\tilde{C}$ is a unit vector). In order for the continuous time and the discrete time models to have the same trajectory (sampled at annual intervals), given the same level of emissions, $C$ is not a unit vector. For the calibration here,

$$
C^{\prime} \approx\left(\begin{array}{llll}
-1.06 \times 10^{-4} & 1.02 & -1.31 \times 10^{-2} & -6.07 \times 10^{-3}
\end{array}\right)
$$

The negative values do not have a physical interpretation. They arise only because I want the continuous time model to "match" the discrete time model. The elements of $C$ sum to 1.
} 


\section{The linear-quadratic model with $n$ tribes}

In the interest of generality, I provide the formulae for the linear-quadratic model that includes an interaction between the control and state variables in the utility function, even though the climate model does not require this interaction. I first make a linear transformation of the state and control variables in order to reduce the dimension of parameter space in the linear-quadratic one-tribe model. I then convert the $n=1$ model to the multi-tribe setting. The next subsections provide formulae for the linear equilibria for general $n$, under both exponentially distributed lifetimes and deterministic lifetimes. I also explain how to obtain nonlinear equilibria for the model with exponentially distributed lifetime. I then discuss the calibration.

The text notes that a specialization of the linear-quadratic problem has the flow payoff quadratic in the control and linear in the state. It is easy to see why there are two globally defined MPE decision rules for this variation, a constant and a linear decision rule. The constant equilibrium decision can be obtained in closed form; to obtain the linear equilibrium it is necessary to solve a quadratic rather than a cubic equation. Nonlinear equilibria must be solved numerically. If agent $i, t$ believes that other agents set their decision to a constant level, agent $i, t$ faces a problem that is linear in the state; the equilibrium decision to that problem is a constant. If agent $i, t$ believes that other agents use a linear control rule, the function $K$ is quadratic in the state; agent $i, t$ therefore has a fictitious control problem that is quadratic in the state; the solution to that problem is a linear control rule.

\section{C.1 Reduction in parameter space}

Here I make a linear transformation of the state and control variables that reduces the dimension of parameter space from 8 to 4 . Begin with a one-tribe model in which the state variable is $\sigma$ and the control variable is $\varphi$. Given the 8 parameters $w, W, v, V, M, G, d, C$, the flow payoff in the one-tribe setting is

$$
-\left(w \varphi+W \varphi^{2}+v \sigma+V \sigma^{2}+M \varphi \sigma\right)
$$


and the equation of motion is

$$
\dot{\sigma}=G+d \sigma+C \varphi
$$

Define a new state and control variable:

$$
\begin{aligned}
S & =\sqrt{2 V}\left(\frac{2 v W-M w}{4 V W-M^{2}}+\sigma\right) \\
X & =\sqrt{2 W}\left(\frac{2 w V-M v}{4 V W-M^{2}}+\varphi\right)
\end{aligned}
$$

With these definitions, the flow payoff and the equation of motion are, respectively,

$$
-\frac{1}{2}\left(X^{2}+S^{2}+m X S\right)+\text { constant, and } \dot{S}=g+d S+c X
$$

with $m \equiv \frac{M}{\sqrt{V W}}$ and

$$
g \equiv \sqrt{2 V}\left(G-D \frac{2 v W-M w}{4 V W-M^{2}}+C \frac{M v-2 w V}{4 V W-M^{2}}\right) \text { and } c \equiv \frac{C \sqrt{V}}{\sqrt{W}} .
$$

The constant in the flow payoff does not affect behavior, so I ignore it henceforth.

\section{C.2 From one tribe to $n$ tribes}

I want to define a flow payoff and an equation of motion (the technology) such that the aggregate feasible payoff does not depend directly on $n$. The equilibrium aggregate payoff and decision rule depends on $n$ only insofar as $n$ alters the equilibrium decision rules of the individual tribes. That is, $n$ has a strategic but not an intrinsic effect on agents' payoffs.

Define the flow payoff and the constraint facing the $i$ 'th tribe as

$$
-\frac{1}{2}\left(\frac{1}{n} S^{2}+n x_{i}^{2}+m x_{i} S\right) \quad \dot{S}=g+d S+c\left(x_{i}+\sum_{j \neq i} x_{j}\right),
$$

where $x_{i}$ is the control variable of the $i$ 'th tribe at an arbitrary point in time. If 
all tribes use the same decision, $x$, the aggregate action is $X=\sum_{i} x_{i}=n x$. The aggregate payoff when all tribes use the same action is $n$ times the first expression in system (45), which equals the first expression in system (43). When all tribes use the same action, the equations of motion in the two systems are obviously identical. The equilibrium flow payoff (where $\left.x=\frac{X}{n}\right)$ simplifies to $-\frac{1}{2 n}\left(S^{2}+X^{2}+m X S\right.$ )

\section{C.3 Calculating the equilibria}

This section provides details for the linear and non-linear equilibria in the model where agents have exponentially distributed lifetime, and for the linear equilibrium where agents have known finite lifetime.

\section{C.3.1 Exponentially distributed lifetime}

For the purpose of obtaining the linear equilibrium under exponentially distributed lifetimes for the two cases, $\lambda<r$ and $\lambda>r$, and for the limiting case $\lambda=\infty$, I introduce constants $\eta, \epsilon, \nu$ that take the values given in Table 5

\begin{tabular}{|l|l|l|l|}
\hline & $\eta=$ & $\epsilon=$ & $\nu=$ \\
\hline if $\lambda>r$ & $\lambda$ & $\theta$ & $\gamma$ \\
\hline if $\lambda<r$ & $\gamma$ & $\lambda-r$ & $\lambda$ \\
\hline if $\lambda=\infty$ & $\mathrm{n} / \mathrm{a}$ & $\epsilon=0$ & $\gamma$ \\
\hline
\end{tabular}

Table 5: values of $\eta, \epsilon, \nu$ for different cases

Here, $\eta$ is the discount rate used in the definition of $K, \epsilon$ is the factor that multiplies the integral in the definition of $-K$, and $\nu$ is the discount rate used in the fictitious control problem. 
The Hamiltonian, $H$, for the fictitious optimal control problem, the necessary conditions for that problem, and the definition of $K$, are

$$
\begin{gathered}
H=-\frac{1}{2}\left(\frac{1}{n} S^{2}+n x^{2}\right)-K(S)+\mu\left(g+d S+c\left(x+\frac{n-1}{n} \Psi(S)\right)\right) \\
\frac{\partial H}{\partial x}=-n x+\mu c=0 \Longrightarrow \mu=\frac{n x}{c}=\frac{X}{c} \\
\dot{\mu}=\nu \mu-\frac{\partial H}{\partial S}=\nu \mu-\left(-\frac{S}{n}-K^{\prime}+\mu\left(d+\frac{n-1}{n} \Psi^{\prime} c\right)\right) \\
K=-\epsilon \int_{0}^{\infty} e^{-\eta t}\left(-\frac{1}{2 n}\right)\left(S^{2}+X^{2}\right) d t .
\end{gathered}
$$

In a symmetric equilibrium, $n x=\Psi \equiv X$.

Differentiating the second equation in system (46) with respect to time and using the third equation gives

$$
\frac{\dot{X}}{c}=\nu \frac{X}{c}-\left(-\frac{S}{n}-K^{\prime}+\frac{X}{c}\left(d+\frac{n-1}{n} c X^{\prime}\right)\right) .
$$

Dividing this equation by $\dot{S}$, using $\frac{\dot{X}}{\dot{S}}=\frac{d X}{d S}=X^{\prime}$, gives

$$
\begin{gathered}
\frac{\dot{X}}{c \dot{S}}=\frac{X^{\prime}}{c}=\frac{\nu \frac{X}{c}-\left(-\frac{S}{n}-K^{\prime}+\frac{X}{c}\left(d+\frac{n-1}{n} c X^{\prime}\right)\right)}{g+d S+c X} \Longrightarrow \\
X^{\prime}=\frac{\left(X n(\nu-d)+S c+c n K^{\prime}\right)}{n(g+d S)-X c(1-2 n)} .
\end{gathered}
$$

The fourth equation in system (46) implies

$$
\begin{gathered}
\eta K=\frac{\epsilon}{2 n}\left(S^{2}+X^{2}\right)+K^{\prime}(g+d S+c X) \Longrightarrow \\
K^{\prime}=\frac{\eta K-\frac{\epsilon}{2 n}\left(S^{2}+X^{2}\right)}{(g+d S+c X)}
\end{gathered}
$$

A MPE must solve the ODEs in the second lines of equations (47) and (48). 
Finding the linear equilibrium In the linear equilibrium, $X$ is a linear and $K$ is a quadratic function of $S$ :

$$
\begin{gathered}
X=a+\Delta S \\
K=\kappa_{0}+\kappa_{1} S+\frac{1}{2} \kappa_{2} S^{2} .
\end{gathered}
$$

The objective is to find the equilibrium values of $a, \Delta$. Substituting these two functions in the ODEs in equations (47) and (48) and equating coefficients in $S$ and 1 for the first equation, and coefficients of $S^{2}$ and $S$ in the second equation, implies

$$
\begin{gathered}
(c-2 c n) \Delta^{2}+n(\nu-2 d) \Delta+c+c n \kappa_{2}=0 \\
(2 d n-n \eta+2 c n \Delta) \kappa_{2}+\left(\epsilon \Delta^{2}+\epsilon\right)=0 \\
(c \Delta+n \nu-d n-2 c n \Delta) a+c n \kappa_{1}-g n \Delta=0 \\
(2 d n-2 n \eta+2 c n \Delta) \kappa_{1}+\left(2 g n \kappa_{2}+2 a \Delta \epsilon+2 a c n \kappa_{2}\right)=0
\end{gathered}
$$

Solving the second equation for $\kappa_{2}$ and substituting the result into the first equation implies

$$
\begin{gathered}
\kappa_{2}=-\frac{\epsilon \Delta^{2}+\epsilon}{2 d n-n \eta+2 c n \Delta} \\
\Sigma \equiv 2 c^{2}(2 n-1) \Delta^{3}+(((8 d-2 \eta-2 \nu) n+(\epsilon+\eta-2 d)) c) \Delta^{2} \\
+\left(\left(\nu \eta-2 d \eta-2 d \nu+4 d^{2}\right) n-2 c^{2}\right) \Delta+c(\epsilon+\eta-2 d)=0 .
\end{gathered}
$$

The correct root of the cubic $\Sigma=0$ must yield a stable equilibrium, i.e. the inequality $d+c \Delta<0$ must hold. Use this root (or roots) to obtain the equilibrium value(s) of $\kappa_{2}$. Using these values, the last two equations in the system (49) are a pair of linear equations in $a, \kappa_{1}$.

Finding nonlinear equilibria I begin by using the argument presented in Appendix B.3, generalized to allow arbitrary values of $\lambda$, but specialized to the linearquadratic setting. Denote $\Phi$ as the value of $X^{\prime}$ at a steady state, i.e. where 
$0=g+d S+c X$. With this definition, $z=d+c \Phi$. A derivation that parallels that which establishes equation (34) gives

$$
\begin{gathered}
K^{\prime}\left(S_{\infty}\right)=\frac{\epsilon}{n}(S+X \Phi)_{\mid \infty} \int_{0}^{\infty} e^{-\eta t} e^{z t} d t= \\
\frac{\frac{\epsilon}{n}(S+X \Phi)_{\mid \infty}}{\eta-z}=\frac{\frac{\epsilon}{n}\left(S-\frac{1}{c}(g+S d) \Phi\right)}{\eta-d-c \Phi} .
\end{gathered}
$$

Substitute this steady state value of $K^{\prime}$ into the steady state value of $X^{\prime}$, using equation (47), and evaluate the result at a steady state $\left(X=-\frac{1}{c}(g+S d)\right)$ to obtain the quadratic in $\Phi$ :

$$
\begin{gathered}
Q \Phi^{2}+L \Phi+C=0 \quad \text { with } \\
Q=(1-n) c(g+d S) \\
L=(d-\eta-\epsilon+n \nu+2 n \eta-3 d n)(g+S d)+g n(d-\eta)+S\left(d^{2} n-d n \eta-c^{2}\right) \\
C=S c(\epsilon+\eta-d)+\frac{n}{c}(g+S d)\left(d \eta-\nu \eta+d \nu-d^{2}\right) .
\end{gathered}
$$

The set of $S$ that can be supported as steady states in a MPE is

$$
\Lambda=\left\{S \mid \exists \Phi \text { for which } d+c \Phi<0 \wedge Q \Phi^{2}+L \Phi+C=0\right\}
$$

To construct a non-linear MPE for this model, pick a value of $S \in \Lambda$ with $X=-\frac{1}{c}(g+S d)$ and solve the pair of ODEs in equations (47) and (48) with these initial conditions. In general, these ODEs must be solved numerically. A possible difficulty arises from the fact that we do not know the domain of such a solution. For example, if we were to solve these ODEs using a function approximation over a domain that is strictly larger than the domain of existence of the ODEs, then the solver might return a poor approximation of the true solution.

MuPad can solve the initial value problem described above; MuPAD's default method is DOPRI78, an embedded Runge-Kutta pair of orders 7 and 8 . This procedure runs into difficulty because the expression for $K^{\prime}$ (and also for $X^{\prime}$ in the case where $n=1$ ) is an indeterminate form at a steady state. In order to 
avoid this difficulty, choose a small $\varepsilon$ and replace the initial condition $\left(S_{\infty}, X_{\infty}, K_{\infty}\right)$ with a nearby point $\left(S_{\infty}+\varepsilon, X_{\infty}+\Phi \varepsilon, K_{\infty}+K^{\prime}\left(S_{\infty}\right) \varepsilon\right)$. This point lies close to the correct boundary, and it lies on the tangent to the trajectory that takes the system to this boundary. The solution to this initial value problem, $X(S)$, can be plotted, to confirm that it does not cross the line $n(g+d S)-X c(1-2 n)$, where $X^{\prime}$ is undefined. (As a consistency check, I confirmed that this approach returns the linear equilibrium when given an initial condition near the steady state to the linear equilibrium.) The results reported in the text use this method of numerically obtaining non-linear equilibria.

In order to calculate the function $\beta$ graphed in Figure 3 I set $d+c \Phi=\tau$, where $\tau$ is a non-positive number close to 0 . For $\tau<0$, the value of $\Phi$ that solves this equation is associated with a stable steady state; $\tau=0$ gives the infimum of values of $\Phi$ associated with a stable steady state. Substituting $\Phi=\frac{-d}{c}$ into $Q \Phi^{2}+L \Phi+C=0$ results in an equation that is linear in $S$. The value of $S$ that solves this equation equals the infimum of $S$ that can be supported as a steady state in a stable MPE. Replacing $S$ with $-\beta \frac{d g}{d^{2}+c^{2}}$ expresses the infimum of stable steady states as a multiple $\beta$ of the UMSS, $-\frac{d g}{d^{2}+c^{2}}$. The resulting equation is linear in $\beta$ and depends on all other parameter values. Setting those values equal to the levels given in Section C.4 results in a $\beta$ as a function of $n, \lambda$ and $\Omega$, shown in Figure 3.

\section{C.3.2 Deterministic lifetime}

The simplicity of the model with exponentially distributed lifetime results from the fact that for that case it is trivial to obtain the ODE for the function $K(S)$. It is not clear how one would obtain the ODE for $K$ in the case where agents have known finite lifetime. I therefore use a slightly different argument to obtain the linear equilibrium in this case, and I leave the non-linear case for future work.

The linear equilibrium $X=a+\Delta S$ must solve the ODE in the second line of equation (47), a fact that leads to the first and the third line of system (49). Under 
the linear policy $X=a+\Delta S$, and given a stock $S(0)=s$,

$$
S(\tau)=\frac{-(g+c a)}{d+c \Delta}\left(1-e^{(d+c \Delta) \tau}\right)+e^{(d+c \Delta) \tau} s .
$$

Substituting this formula into the flow payoff $U=-\frac{1}{2 n}\left(S^{2}+(a+\Delta S)^{2}\right)$ and collecting terms,

$$
\begin{gathered}
U=\sigma_{2} s^{2}+\sigma_{1} s+\sigma_{0} \text { with } \\
\sigma_{2}=-\frac{1}{2 n}\left(e^{2((d+c \Delta) \tau)}+\Delta^{2} e^{2((d+c \Delta) \tau)}\right) \\
\sigma_{11}=-\frac{1}{2 n}\left(-2 \frac{c}{d+c \Delta}\left(1-e^{(d+c \Delta) \tau}\right) e^{(d+c \Delta) \tau}+2\left(1-c \frac{\Delta}{d+c \Delta}\left(1-e^{(d+c \Delta) \tau}\right)\right) \Delta e^{(d+c \Delta) \tau}\right) \\
\sigma_{12}=-\frac{1}{2 n}\left(-2 \frac{g}{d+c \Delta}\left(1-e^{(d+c \Delta) \tau}\right) e^{(d+c \Delta) \tau}-2 \Delta^{2} \frac{g}{d+c \Delta}\left(1-e^{(d+c \Delta) \tau}\right) e^{(d+c \Delta) \tau}\right) \\
\sigma_{1}=a \sigma_{11}+\sigma_{12} \\
\sigma_{0}=-\frac{1}{2 n}\left(\frac{(-g-c a)^{2}}{(d+c \Delta)^{2}}\left(1-e^{(d+c \Delta) \tau}\right)^{2}+\left(a+\Delta \frac{-g-c a}{d+c \Delta}\left(1-e^{(d+c \Delta) \tau}\right)\right)^{2}\right)
\end{gathered}
$$

Under the linear policy, $K(s)$ is a quadratic function: $K(s)=\kappa_{2} s^{2}+\kappa_{1} s+\kappa_{0}$. This fact and the definition of $K$ imply

$$
\kappa_{2} s^{2}+\kappa_{1} s+\kappa_{0}=(r-\lambda) \int_{0}^{T} \frac{(T-\tau)}{T}\left(e^{-r \tau} \sigma_{2} s^{2}+\sigma_{1} s+\sigma_{0}\right) d \tau
$$

Equating coefficients gives

$$
\begin{aligned}
& \kappa_{2}=(r-\lambda) \int_{0}^{T} \frac{(T-\tau)}{T} e^{-r \tau} \sigma_{2} d \tau \\
& \kappa_{1}=(r-\lambda) \int_{0}^{T} \frac{(T-\tau)}{T} e^{-r \tau} \sigma_{1} d \tau
\end{aligned}
$$

The first line of system (49), together with the definitions of $\kappa_{2}$ and $\sigma_{2}$ is a nonlinear equation in $\Delta$. A solution that satisfies $d+c \Delta<0$ is a stable root. Given a numerical value for $\Delta$, the third line of system (49), together with the definitions of $\kappa_{1}$ and $\sigma_{1}$ imply

$$
a=-n \frac{c \sigma_{14}-g \Delta}{c n \sigma_{13}+c \Delta+n \lambda-d n-2 c n \Delta}
$$




\section{C.4 Calibration of the climate model}

I first express the model in "natural units" and then rewrite the model so that the control and state variables are percentages that have a convenient interpretation. I then use the transformation in Appendix C.1 to reduce the dimension of parameter space.

Let $Y_{t}$ be the stock variable at time $t$, ppm of carbon and $x$ be the flow, measured in ppm per year. (One ppm by volume equals 2.13 GtC.) The equation of motion for the stock of greenhouse gases is

$$
\dot{Y}=\bar{g}+\delta Y+x
$$

The parameters $\bar{g}, \delta$, and 1 on the right side correspond to $G, d, C$ in equation (41); $Y$ corresponds to $\sigma$ and $x$ corresponds to $\varphi$. To calibrate the model, I set the half life of carbon to 83 years, the steady state in the absence of anthropogenic emissions equal to the pre-industrial level to $280 \mathrm{ppm}$, and assume that under BAU the stock increases from the current level, 380, to 700 in 90 years. These assumptions imply

$$
\delta=-8.3511709 \times 10^{-3}, \quad \bar{g}=2.3383278, \quad x^{\text {bau }}=5.8926860 .
$$

With these parameters, the steady state stock under BAU is $986 \mathrm{ppm}$, and after 200 years of BAU the stock reaches $872 \mathrm{ppm}$.

For ease of interpretation, it is convenient to express the control variable, $A$, as abatement as a percent of BAU emissions, and the stock, $s$, as the percent increase over preindustrial levels:

$$
A=\frac{x^{\text {bau }}-x}{x^{\text {bau }}} 100 \text { and } s=\frac{Y-280}{280} 100
$$

With these definitions and the parameter values in equation (56), the equation of motion is

$$
\dot{s}=\delta s-\frac{1}{280} x^{\mathrm{BAU}} A+\left(\frac{5}{14} x^{\mathrm{BAU}}+100 \delta+\frac{5}{14} \bar{g}\right)=d s+C A+G
$$


with

$$
G=2.1045307 \quad d=\delta=-8.3511709 \times 10^{-3} \quad C=-2.1045307 \times 10^{-2} .
$$

In this stationary setting, denote the constant $Z$ as gross world product (GWP) exclusive of climate related damage and abatement costs. In this linear-quadratic model, the flow cost of abatement, as a percent of GWP, and the flow cost of the stock, as a percent of GWP are, respectively,

$$
\frac{b}{2} \frac{A^{2}}{Z} 100 \text { and } \frac{h}{2} \frac{s^{2}}{Z} 100
$$

For calibration, suppose that the flow cost of a $50 \%$ reduction in emissions relative to BAU $(A=50)$, is $Q$ percent of GWP; and the flow cost of doubling of ppm relative to pre-industrial level $(s=100)$, is $P$ percent of GWP. These assumption imply

$$
b=\frac{1}{125000} Z Q, \quad h=\frac{1}{500000} P Z \quad \Longrightarrow \frac{h}{b}=\frac{P}{4 Q}
$$

Define $\Omega=\frac{P}{Q}$, so that the total flow costs, as a percent of GWP, corresponding to actual abatement $A$ and actual stock $s$ equal

$$
\left(\frac{b 100}{2 Z}\right)\left((A)^{2}+\frac{\Omega}{4}(s)^{2}\right) .
$$

Hereafter I drop the positive factor $\frac{b 100}{2 Z}$ to write flow benefits (negative costs) as

$$
-\left(A^{2}+\frac{\Omega}{4} s^{2}\right)
$$

Table 6 shows the correspondence between the variables and parameters in the general model in Section C.1 and in the climate model here.

\begin{tabular}{|l|l|l|l|l|l|l|l|l|l|l|}
\hline & control & state & & & & & & & & \\
\hline general & $\varphi$ & $\sigma$ & $w$ & $W$ & $v$ & $V$ & $M$ & $G$ & $d$ & $C$ \\
\hline climate & $A$ & $s$ & 0 & 1 & 0 & $\frac{\Omega}{4}$ & 0 & $G$ & $d$ & $C$ \\
\hline
\end{tabular}


Table 6: Correspondence between parameter values in general model from Section C.1 and the climate model in this section

Equation (58) gives the numerical values of $G, d, C$ that appear in the last row of Table 6, for this calibration. Use Table 6, the numerical values in equation (58) and the formulae in equation (44), to obtain the values of the model parameters that are used in finding the equilibria in the climate model:

$$
g=1.4881279 \sqrt{\Omega} \text { and } c=-1.0522654 \times 10^{-2} \sqrt{\Omega}
$$

In interpreting the equilibrium results, it is important to keep in mind that the model is solved in terms of the transformed state and control $S$ and $X$. The percent increase in the stock relative to preindustrial level, $s$, and aggregate abatement as a percent of BAU emissions, $A$, are related to $S, X$ using system (42) and the correspondences in Table 6:

$$
s=\sqrt{\frac{2}{\Omega}} S, \text { and } A=\frac{X}{\sqrt{2}} .
$$

The initial condition for the problem is $s=\frac{380-280}{280} 100=35.714286$ or $S=35$. $714286 \sqrt{\frac{\Omega}{2}}$. The text describes the results in terms of the stock of atmospheric carbon expressed in ppm. 